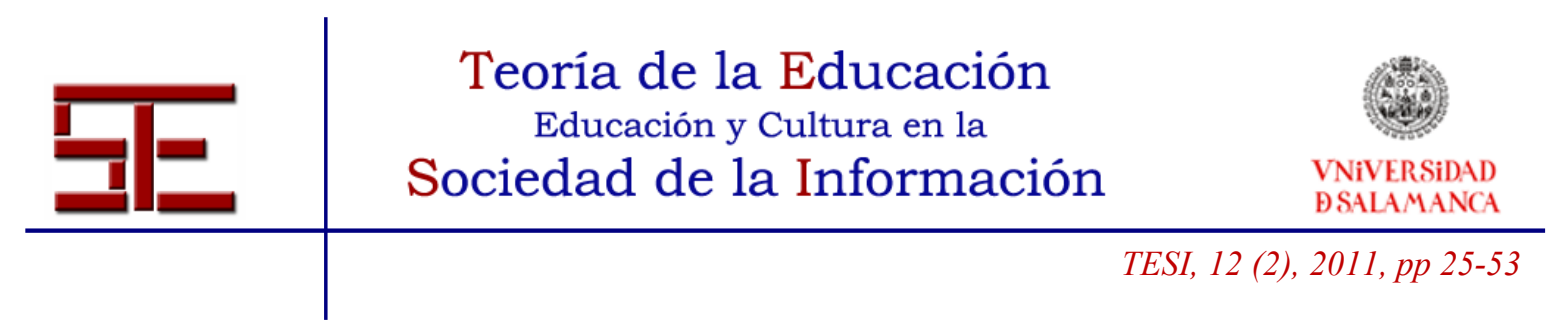

\title{
BRECHA DIGITAL DE GÉNERO: UNA REVISIÓN Y UNA PROPUESTA
}

Resumen: En este artículo ofrecemos una revisión de los estudios sobre la brecha digital de género a nivel nacional e internacional, centrándonos concretamente en las investigaciones sobre la infrarrepresentación de las mujeres en el ámbito formativo y profesional de las nuevas tecnologías de la información y la comunicación (TIC). Asimismo, caracterizamos brevemente las intervenciones e iniciativas desarrolladas en ese ámbito cuyo objetivo ha sido contrarrestar esta situación. Después, evaluamos críticamente los supuestos teóricos de esas investigaciones e intervenciones, demasiado centradas en considerar la cuestión un problema "de las mujeres", para, finalmente, ofrecer recomendaciones que vayan en la dirección de articular una perspectiva de género que nos permita avanzar en el conocimiento de las causas concretas y las posibles soluciones de la brecha digital de género sin esencializar ni el género ni la tecnología.

Palabras clave: Brecha digital; Género; Estudios Sociales de la Ciencia y la Tecnología.

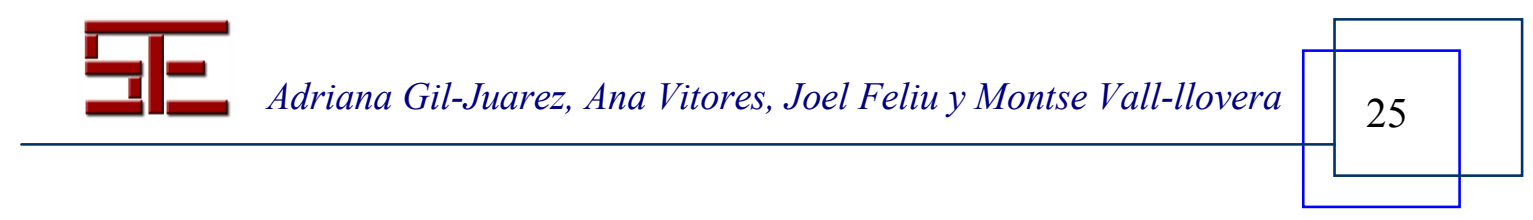




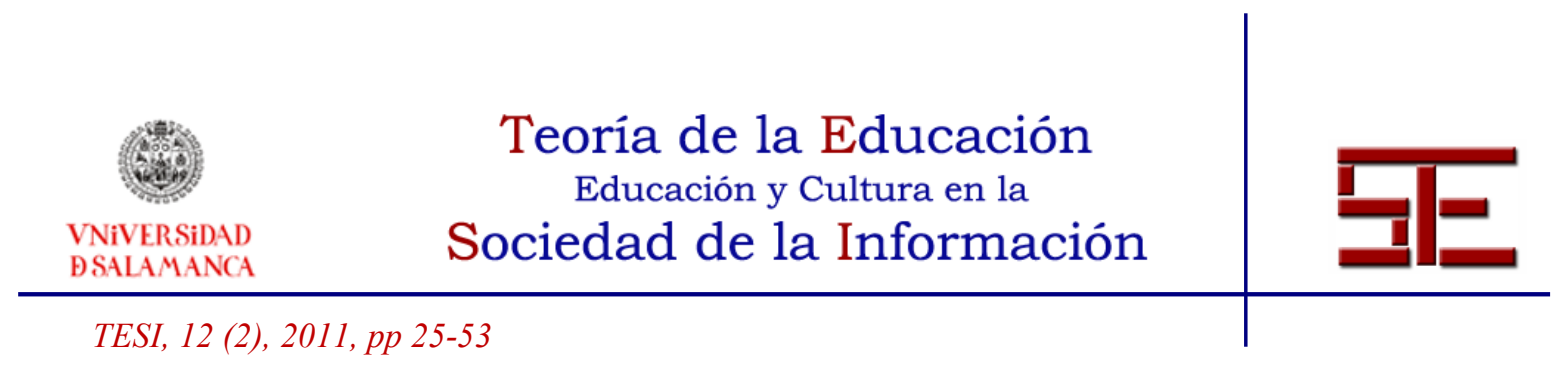

GENDER DIGITAL DIVIDE: A REVIEW AND A PROPOSITION

\begin{abstract}
In this article we offer a review of national and international studies of the gender digital divide, centering specifically in the research on women underrepresentation in the educational and professional field of new information and communication technologies (ICT). We also briefly characterize the interventions and actions developed in this field whose aim has been to counter this situation. Afterwards, we evaluate critically the theoretical assumptions of the reviewed investigations and undertaken actions, too centered in considering this question "a women's problem". Finally, we offer some recommendations to articulate a gendered perspective, which would allow us to progress in the knowledge of the concrete causes and the possible solutions of the gender digital divide without essentializing neither gender nor technology.
\end{abstract}

Keywords: Digital divide; Gender; Social Studies of Science and Technology.

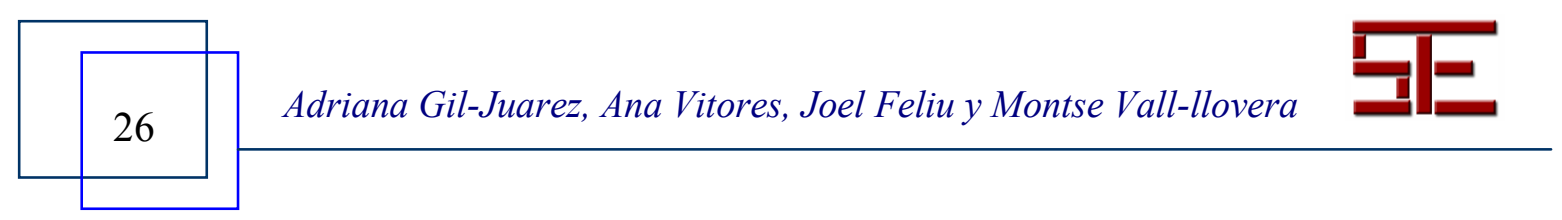




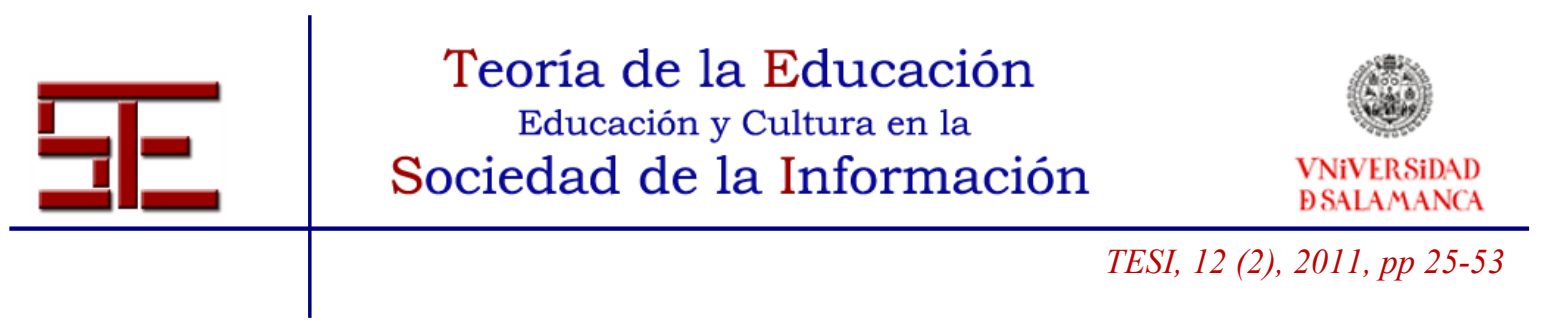

\section{BRECHA DIGITAL DE GÉNERO: UNA REVISIÓN Y UNA PROPUESTA}

Fecha de recepción: 22/04/2011; fecha de aceptación: 01/06/2011; fecha de publicación: 27/07/2011

Adriana Gil-Juarez

adriana.gil@urv.cat

Universitat Rovira y Virgili

Ana Vitores

anna.vitores@uab.cat

Universitat Autónoma de Barcelona

Joel Feliu

joel.feliu@uab.cat

Universitat Autónoma de Barcelona

Montse Vall-llovera

mvall-llovera@uoc.edu

Universitat Oberta de Catalunya

\section{1.- LAS MUJERES EN LAS TIC}

La última década ha visto como se consolidaba la llamada brecha digital de género como objeto de estudio. Puede parecer paradójico que esta preocupación por las causas y las consecuencias de una supuesta fractura digital se consolide precisamente en una década que ha visto como múltiples estudios de los países occidentales han encontrado algo aparentemente opuesto: un incremento del número de mujeres que acceden a las nuevas tecnologías de la información y la comunicación (TIC en adelante). Más aún, ¿por qué debería existir siquiera una preocupación hacia algo que aparentemente pertenece al pasado? Si hombres y mujeres ya acceden en pie de igualdad a las TIC y si ellas lo hacen en un número cada vez mayor, dicha brecha digital, de existir, estaría cerrándose, con lo cual no deberíamos perder un minuto investigándola. Sin embargo, precisamente este paradójico incremento de estudios sobre la brecha digital, cuando ésta supuestamente se estaría cerrando, ha permitido visibilizar un hecho clave: la brecha digital de género ya no es un problema ligado al simple acceso y uso de las TIC, persiste y se agrava a pesar de accesos y usos cada vez mayores. Por ello en este artículo mostraremos como ésta es una brecha existente que perdura y que se agrava; revisaremos los estudios que la describen; las intervenciones que se han planteado para

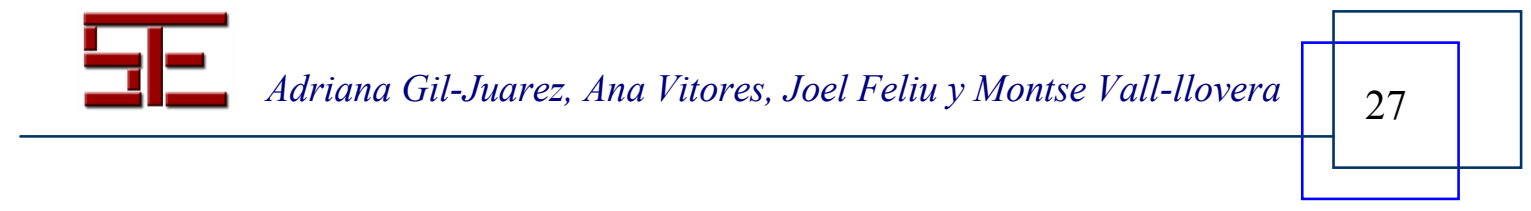




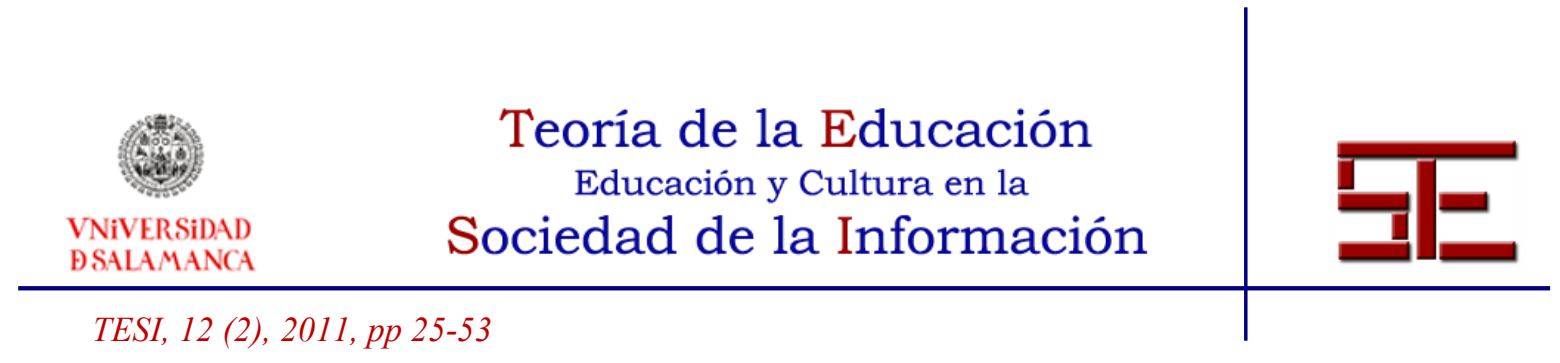

frenar y/o revertir su ensanche; e intentaremos proponer una mirada alternativa a las habituales con la finalidad de conseguir formular las estrategias más adecuadas para su cierre.

Como señala Cecilia Castaño, la brecha digital de género "está relacionada con el dominio masculino de las áreas estratégicas de la educación, la investigación y el empleo relacionado con las ciencias, las ingenierías y las TIC" (Castaño, 2008,10). No es extraño, pues, que la investigación sobre la brecha digital de género haya identificado el problema de las "mujeres $y$ las TIC" como un problema de "las mujeres en las TIC" (Faulkner y Lie, 2007). Es decir, el grueso de los estudios sobre género y TIC se ha centrado en documentar la inferioridad numérica de las mujeres en el ámbito de los estudios, la investigación y la profesión de las TIC.

En este marco, dentro de la literatura académica, se ha hecho habitual referirse a la presencia de las mujeres en el campo de las TIC recurriendo a la imagen de una "tubería" que se "estrecha" o "que pierde" (shrinking pipeline o leaky pipeline) (Adams et. al., 2003; Blickenstaff, 2005; Camp, 1997; Metcaf, 2010; Varma y Hahn, 2008). La mayoría de los estudios destacan que estamos ante una tubería que pierde gente a lo largo de las distintas etapas de vida formativa y profesional de las mujeres de este campo y, además, a lo largo de los años: refiriéndose con esta imagen al hecho de que a medida que ellas se internan en este terreno se multiplican las ocasiones para abandonarlo. De hecho, a medida que el sector se profesionaliza y se especializa cada vez hay menos presencia de mujeres.

De este modo, la "tubería" de las TIC empezaría a estrecharse para las mujeres ya en el bachillerato, durante el cual las chicas optan en proporciones más bajas por las asignaturas y/o los perfiles tecnológicos (Eidelman y Hazzan, 2006; Margolis et al., 2003). Así, por ejemplo, en 2009, en Estados Unidos, las mujeres representaban el 56\% de las personas que se presentaron al llamado examen de Colocación Avanzada (Advanced Placement exam), pero fueron sólo el 18\% de las personas que realizaron el examen de Colocación Avanzada en Informática (CS AP, Computer Science Advanced Placement exam) (National Center for Women \& Information Technology [NCWIT], 2010). De hecho, desde 1999 el Programa de Colocación Avanzada en Informática es sistemáticamente el programa en el que menos mujeres hay (NCWIT, 2010). En el Reino Unido, en 2008, sólo el 10\% de las personas que optaron por las asignaturas de informática en la educación secundaria eran mujeres (BCS and eSkills, 2009). La tendencia es parecida en países como el nuestro, donde también es bajo (3\%) el

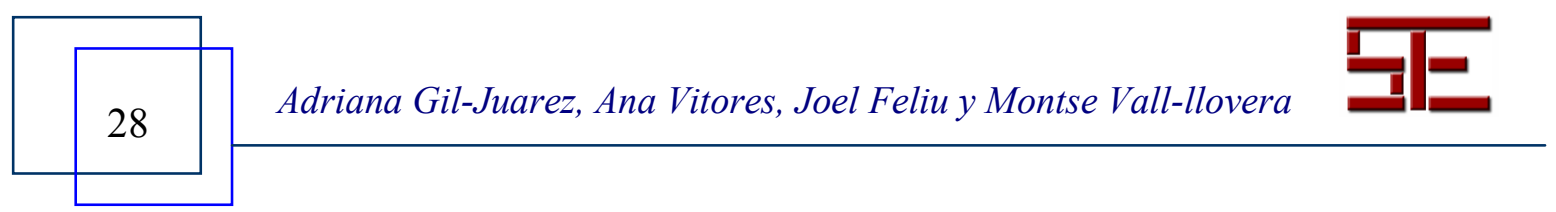




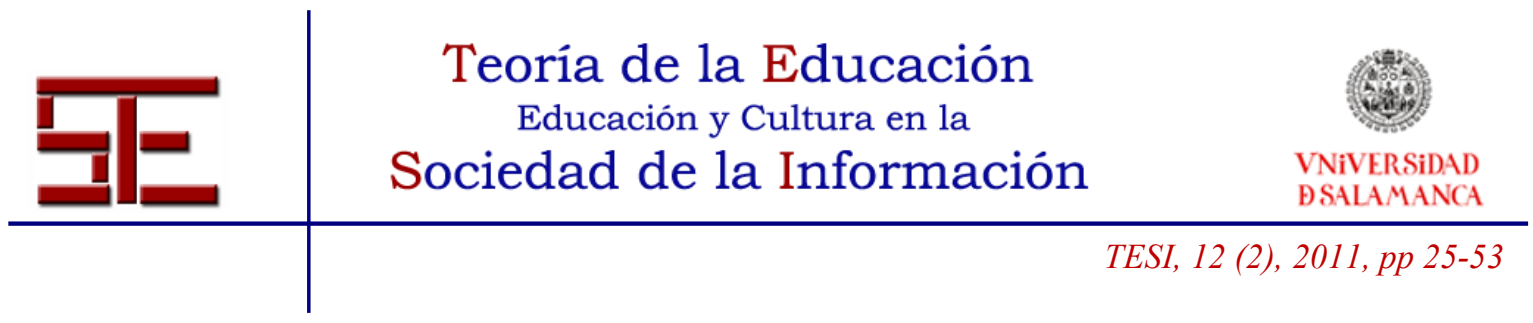

porcentaje de mujeres que cursa el itinerario tecnológico en bachillerato (Sáinz y López Saénz, 2010).

La infrarrepresentación de las mujeres en el mundo TIC se hace especialmente visible en la siguiente etapa, la de la formación universitaria. Así, aunque casi de forma generalizada se constata que cada vez se matriculan más mujeres en las universidades de todo el mundo ( $\mathrm{y}$, de hecho, el número de mujeres que se matriculan en las universidades es superior al número de hombres tanto en los países de la Unión Europea [Eurostat, 2009] como en los Estados Unidos [NCWIT, 2010], es evidente que existe todavía un contundente sesgo de género en relación a los estudios por los que se opta. En efecto, las mujeres son claramente minoría dentro del ámbito técnico y, dentro de este ámbito, son especialmente preocupantes los datos relativos a la informática (ingeniería informática, ciencias de la computación o tecnologías de la información, en función de la denominación de los estudios en distintos países). En países aparentemente tan diferentes como Alemania (Black et. al., 2005); Australia (Anderson et al., 2008); Canadá (Ross, 2010); China (Black et. al., 2005); Estados Unidos (Cohoon y Aspray, 2006); Inglaterra (BCS \& eSkills, 2009); Italia (Black et. al., 2005); Israel (Gal-Ezer, et al., 2009); o Suecia (Boivie, 2010) se reseña un descenso de mujeres matriculadas en informática en los últimos años. Así, por ejemplo, en Estados Unidos la informática (computer science) es la única de las llamadas disciplinas en el ámbito STEM (acrónimo en inglés de Science, Technology, Engineering and Math, es decir Ciencia, Tecnología, Ingeniería y Matemáticas) en la que la representación de las mujeres a nivel de licenciatura/grado (bachelor's level) ha sufrido un descenso. Y un descenso significativo: en 1985 un 37\% de las personas licenciadas en informática en Estados Unidos fueron mujeres, porcentaje que descendió a un 28,4\% en 1994 (Camp, 1997). Y la tendencia a la baja parece lejos de reconducirse, en 2008 sólo un $18 \%$ de las personas que obtuvieron títulos en el campo de la informática eran mujeres (NCWIT, 2010).

Nuestro entorno más inmediato no es una excepción: también en las universidades del Estado español las mujeres son claramente minoría en los estudios en el ámbito de las TIC (Córdoba y Jofre, 2009; Pérez et. al., 2003; Fernández et. al., 2008, Sanz, 2008). $\mathrm{Y}$, siguiendo la tendencia internacional señalada, la infrarrepresentación resulta especialmente insidiosa en el campo de la informática (Gómez Ferri, 2004; Pérez Fuentes et al., 2003; Sanz, 2005, 2008). Así, aunque en el curso académico 2008-2009 las mujeres representaban el $54 \%$ de las personas matriculadas en la universidad, sólo

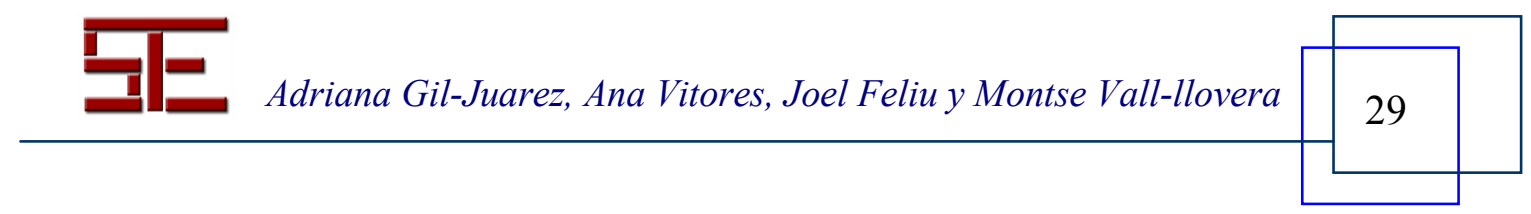




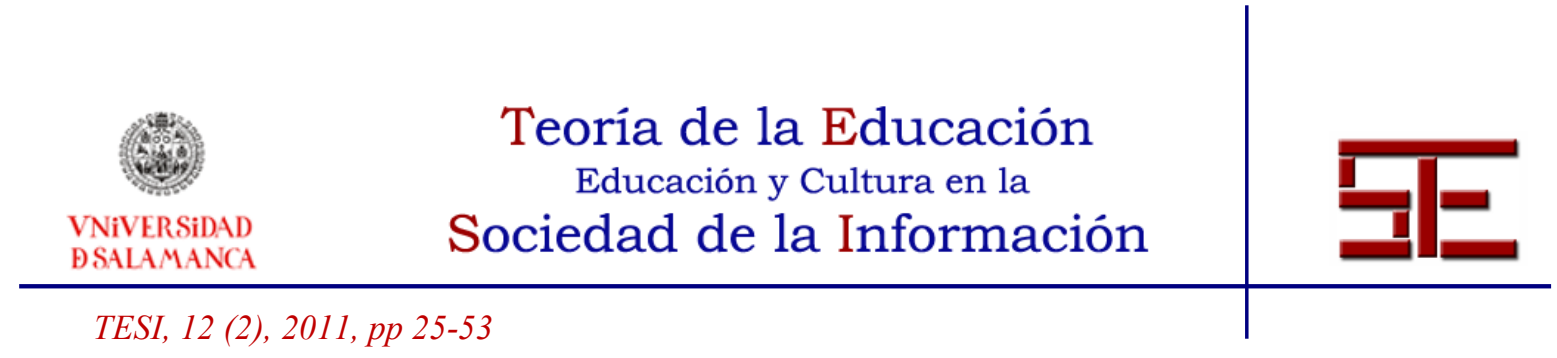

un $17 \%$ de las personas matriculadas en los estudios de informática eran mujeres (Sáinz; López-Sáez, 2010).

También es escaso el número de mujeres que acceden a profesiones en el sector de las TIC según muestran los datos de países como Australia (Craig, 2006); Austria (OCDE, 2007); Bélgica (Valenduc et al., 2004); Bulgaria (Seybert, 2007); Estados Unidos (OCDE, 2007; Simard y Shannon, 2010; Ashcraft y Blithe, 2009); Francia (Valenduc et. al., 2004); Luxemburgo (OCDE, 2007); los Países Bajos (Seybert, 2007); el Reino Unido (Glover y Guerrier, 2010; Griffiths y Moore, 2010) o Israel (Kark, 2007). Y, de nuevo, la infrarrepresentación, lejos de corregirse, parece agudizarse con el paso del tiempo: podemos tomar como ejemplo los datos del Reino Unido, donde las mujeres pasaron de representar el $25 \%$ de la fuerza de trabajo del sector TIC en 1997 a un 23\% el 2008 (Glover y Guerrier); o el ejemplo de Estados Unidos, donde en 1991 las mujeres representaban el 36\% de la fuerza de trabajo en el sector de las TIC, porcentaje que ha descendido a un 25\% en 2009 (Ashcraft y Blithe, 2009).

$\mathrm{Si}$ atendemos al detalle en profesiones concretas dentro del sector TIC desde el año 2000 hasta el 2009, en Estados Unidos, sólo el porcentaje de mujeres trabajando en la categoría Network Systems \& Data Communications Analysts se ha mantenido estable $(25 \%)$, en el resto de perfiles ha descendido el porcentajes de mujeres (por ejemplo, para la categoría computer programers el porcentaje era de un $26 \%$ en 2000 y de un $20 \%$ en 2009; para la categoria "computer software engineers" el porcentaje pasó de un $24 \%$ [2000] a un 20\% [2009] y para el perfil computer hardware engineers se pasó de un $22 \%$ de mujeres [2000] a un 9\% [2009] [NCWIT, 2010]. En este mismo contexto, el problema no es sólo atraer a mujeres al trabajo en el sector de las TIC: la tubería también se estrecha en este último tramo. Así, en Estados Unidos, un 56\% de las mujeres que trabajan en empresas en el campo de las TIC abandonan sus puestos a la mitad de su carrera (10-20 años); en una proporción que dobla el porcentaje de hombres que abandonan las empresas TIC (Simbard y Shannon, 2010).

Los datos ofrecidos hasta ahora nos muestran un fenómeno especialmente "anómalo" en la historia de las profesiones, y nos permiten entender que investigadoras como Caroline Clarke se vean llevadas al extremo de afirmar que si la tendencia continúa al ritmo en que lo ha hecho en el período que va de 1986 a 2006, "no habrá ninguna mujer licenciada en informática en 2032" (Clarke, 2010, 27).

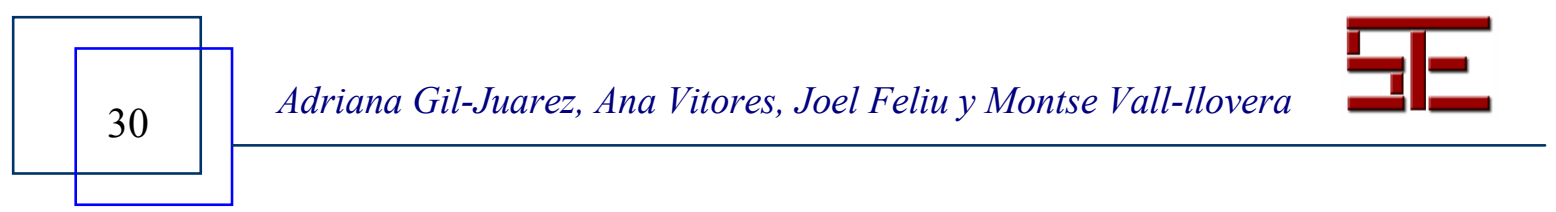




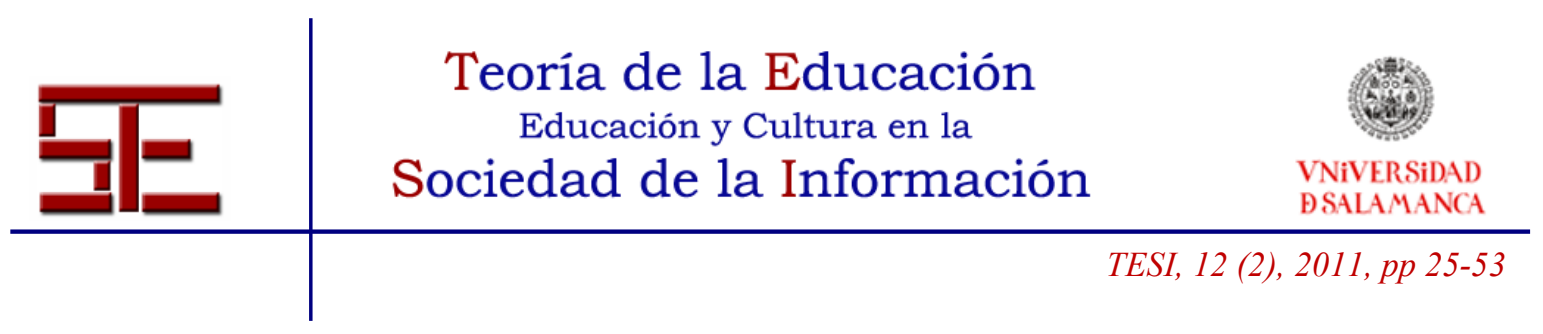

\section{1.- Más mujeres en las TIC: intervenciones planteadas}

En este panorama, el interrogante que se plantea la investigación académica es claro y constante en los últimos años: ¿por qué hay tan pocas mujeres en el ámbito de las TIC? La pregunta, como hemos visto por los datos, se formula en especial para el ámbito de la informática, como muestra, obsérvense los sucintos títulos de los siguientes trabajos académicos: Why are There so Few Female Computer Scientists? (Spertus, 1991) Why women avoid computer science (De Palma, 2001); Why Are So Few Women in Computer Science?"; (Beyet et.al., 2002); Why are there so few women in information technology? (Rosenbloom et al., 2008); Technology majors: why are women absent? (Harris et al., 2009); Computer Science: Where (and why) have all the women gone? (Ross, 2010), Why Aren't More Women in Computer Science? (Whitecraft y Williams, 2011). Una pregunta que se torna insidiosa si tenemos presente que este papel minoritario y en declive de las mujeres en la informática corre paralelo a, cuando no viene precedido por, años de intervenciones diseñadas no sólo para aumentar el papel de las mujeres en las TIC, sino específicamente para aumentar el número de chicas que acceden a estudios superiores en informática y TIC. Como sintetizan Joanne McGrath Cohoon y William Aspray (2006), "tenemos que enfrentarnos al hecho de que veinticinco años de intervenciones no han funcionado" (2006, ix).

En efecto, durante esos veinticinco años las intervenciones han sido constantes y variadas, y todas ellas han tenido como objetivo prioritario incrementar el número de mujeres en las TIC. Son múltiples los sistemas de clasificación de las mismas (AAUW, 2000; Klawe et al., 2009; Miliszewska y Moore, 2010; Misa, 2010; Sorenson, 2002): así, por ejemplo, se clasifican en función del lugar del recorrido de "la tubería que pierde" en el que intervienen (educación primaria o secundaria, educación superior o sector empresarial), en función del tipo de organización implicada en el diseño y la ejecución de las iniciativas (administraciones públicas, instituciones educativas, grupos empresariales, asociaciones o grupos independientes...), en función del público al que se dirige (chicas, profesores/as, maestros/as, padres y madres, profesionales...) o en función del tipo de actividades desarrolladas (talleres, cursos, cambios en el currículum, campañas institucionales, creación de plataformas de apoyo, grupos de trabajo...). Sin lugar a dudas, la clasificación más informadora tiene que ver con las acciones específicas que desarrollan estas intervenciones para conseguir incrementar el número de mujeres en las TIC (Chinn y VanDeGrift, 2008; Cuny y Aspray, 2000; Klawe et al., 2009; McGrath, 2001; Yelland et al., 2002):

- Exponer experiencias y modelos femeninos en el ámbito de las TIC a

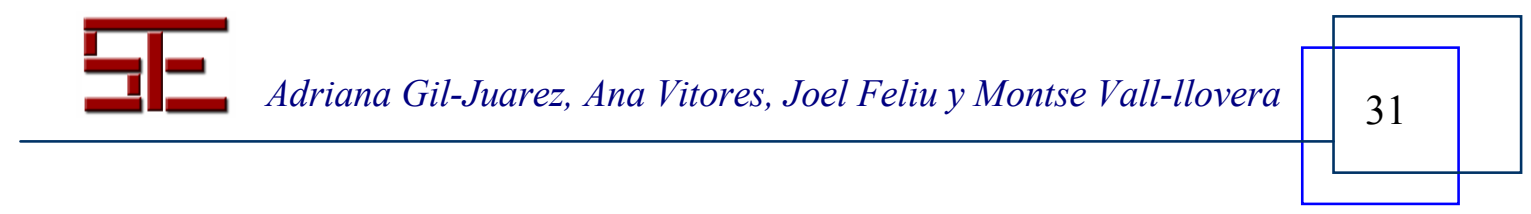




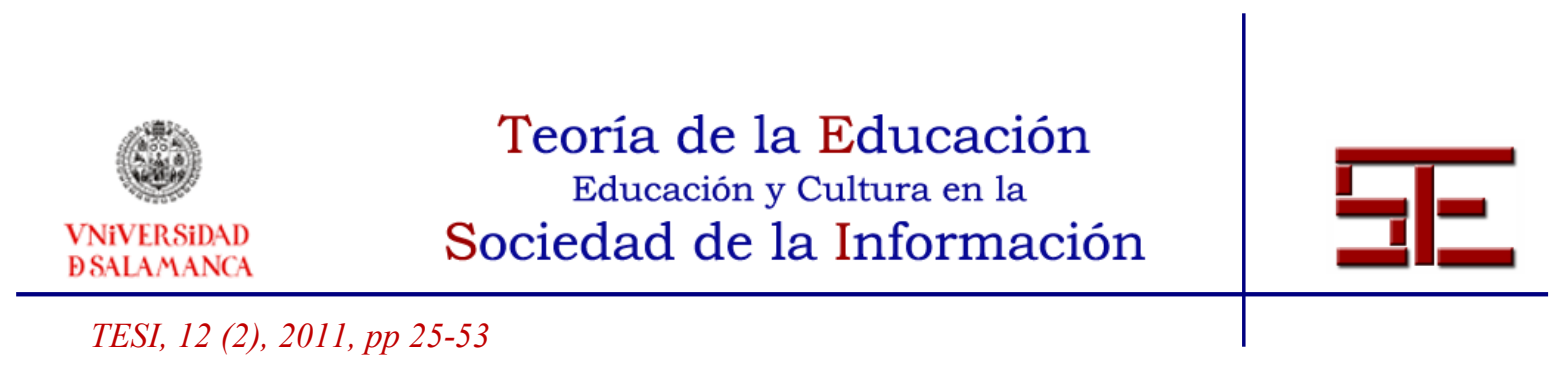

niñas y jóvenes .

- Facilitar herramientas, currículos y métodos de enseñanza de las TIC "amigables" para las niñas y las jóvenes.

- Cambiar la imagen pública de la informática, en especial romper con los estereotipos masculinos asociados a la profesión.

- Trabajar con el profesorado de los distintos niveles educativos para implicarlo en la divulgación y el enrolamiento en el campo de la Informática.

- Crear programas de tutorización, o promover políticas diversas de captación y mantenimiento de mujeres en el entorno académico y/o laboral de las TIC.

De entre este tipo de intervenciones sobresalen las estrategias focalizadas en el reclutamiento temprano. Medidas que encuentran su ejemplo paradigmático en los llamados girls' day, unas jornadas que se vienen desarrollando en EEUU y en diversos países europeos, España incluida, dirigidas a dar a conocer a las chicas de los institutos de secundaria y bachillerato la profesión y los estudios tecnológicos que, además, acostumbran a contar con la colaboración de mujeres profesionales, especialistas en el campo, para ofrecer modelos con los que identificarse.

$\mathrm{Si}$, como hemos comentado, los objetivos de estas intervenciones son especialmente informativos es porque se basan en buena medida en la respuesta dada a la pregunta que plantea la investigación actual “¿por qué hay tan pocas mujeres en las TIC?”. En efecto, aunque estas respuestas son variadas, y contienen énfasis distintos en función del marco disciplinar del que se parte, es posible encontrar en ellas un conjunto de explicaciones especialmente recurrentes, imaginadas en términos de "barreras de acceso": la falta de modelos femeninos de referencia en el ámbito, los estereotipos masculinos y generalmente negativos (la imagen del "nerd") asociados a la informática, y las dificultades y la hostilidad del ambiente académico y laboral en el campo de las TIC para las mujeres (Ahuja, 2002; Cohoon y Aspray, 2006; Gürer y Camp, 2002; Clarke, 2010; Valenduc et al., 2004). La imagen de la "barrera" ha sido recurrente en las investigaciones que hemos presentado hasta ahora y ha conducido y orientado la mayor parte de las intervenciones realizadas hasta el momento. Sin embargo, estas intervenciones no han conseguido marcar un cambio de tendencia en el decremento de la presencia de mujeres en el ámbito de las TIC, lo que significa que seguramente nos hallamos ante un problema mucho más complejo, que va más allá de la ausencia de

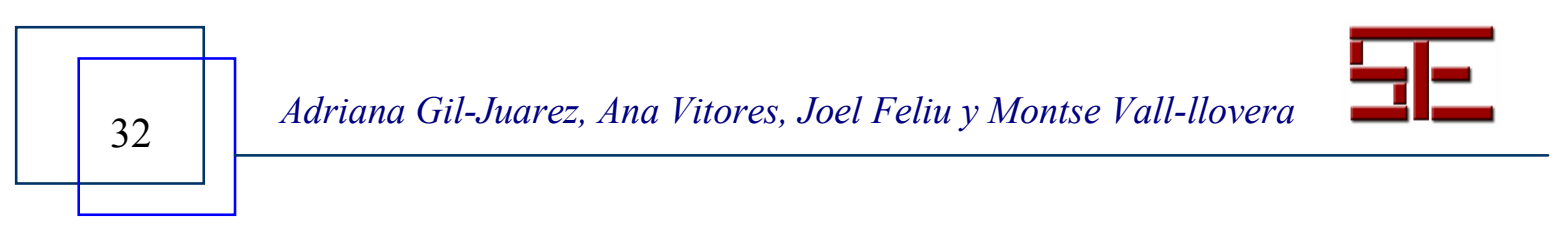




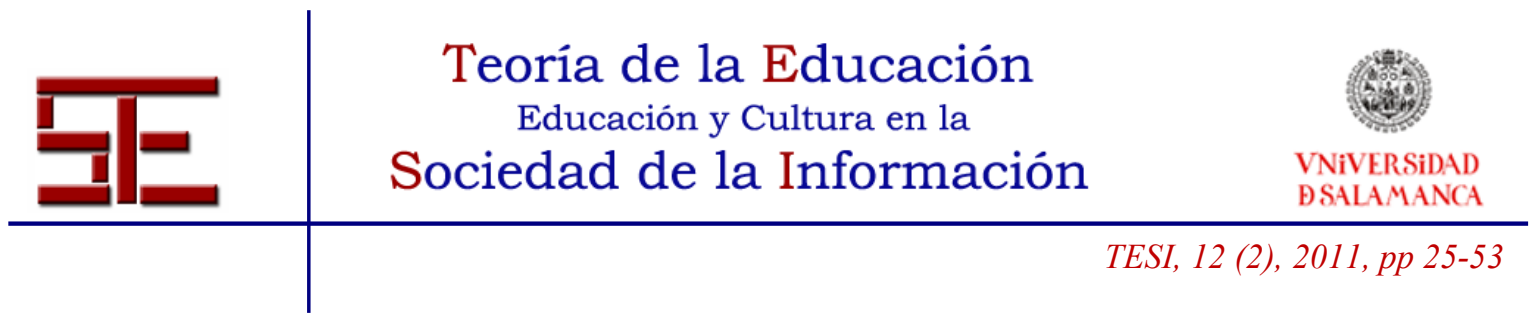

modelos femeninos y la presencia de estereotipos, prejuicios y prácticas discriminatorias.

\section{2.- LAS MUJERES Y LAS TIC}

Las barreras concretas no aparecen en el vacío social sino en marcos de significado; es justamente porque tienen sentido que adquieren realidad. En el caso que nos ocupa, el sentido es la relación diferencial con las TIC que se establece en función del género. Como hemos indicado al inicio, los estudios reseñan que las mujeres son usuarias de las TIC en cifras cada vez más parecidas a las de los hombres, e incluso superiores en lo que respecta a algunas tecnologías, como los móviles o las redes sociales (Booth et al., 2010; Brynin, 2006; Sorenson, 2002). Sin embargo, aunque el acceso se iguale entre hombres y mujeres, se mantienen o incluso crecen las desigualdades en cuanto a los usos y los conocimientos de diferentes tipos de TIC; las habilidades necesarias para acceder a información, los conocimientos y la formación mediante las TIC; y finalmente, en las habilidades para vivir y trabajar en entornos vertebrados por esas mismas TIC (Sáinz et al., 2008).

De este modo, aunque en la década de 1990 se apuntaba al acceso diferencial a las TIC como una de las barreras que explicaban la escasa presencia de mujeres en las TIC (Gürer y Camp, 2002), en los últimos años se ha hecho evidente que, una vez superada esa primera barrera, se observa una "segunda brecha digital" (Castaño, 2008, 2009; Castaño et al., 2009; Gargallo-Castel, et al., 2010). Así, la Primera Brecha Digital remite al acceso desigual generalizado a las TIC, a menudo concretada como la brecha entre aquellas personas que tienen y utilizan ordenadores y tienen conexión a Internet (y la calidad de la misma) y aquellas que no. Ésta ha sido una brecha estudiada y expresada en términos básicamente cuantitativos. En cambio, el concepto de Segunda Brecha Digital nos remite a unas desigualdades en unos términos (por ejemplo, la intensidad, los usos, las habilidades, la soltura o la afección respecto a las TIC) que no es posible operacionalizar únicamente en términos cuantitativos. Y es esta segunda brecha digital la que explica la desigual relación que hombres y mujeres establecen con las TIC y, en última instancia, la distancia que muchas mujeres toman respecto del uso experto de las nuevas tecnologías. El concepto de uso experto toma especial importancia en relación al acceso a los estudios superiores, dado que solamente aquellas que tengan interés en desarrollar una práctica experta accederán a dichos estudios. Esa distancia respecto al uso experto, se presenta vinculada al interés y la afección hacia las TIC. En este sentido, los estudios señalan que chicos y hombres tienen sentimientos más positivos hacia los ordenadores, así como que niñas y mujeres se sienten menos

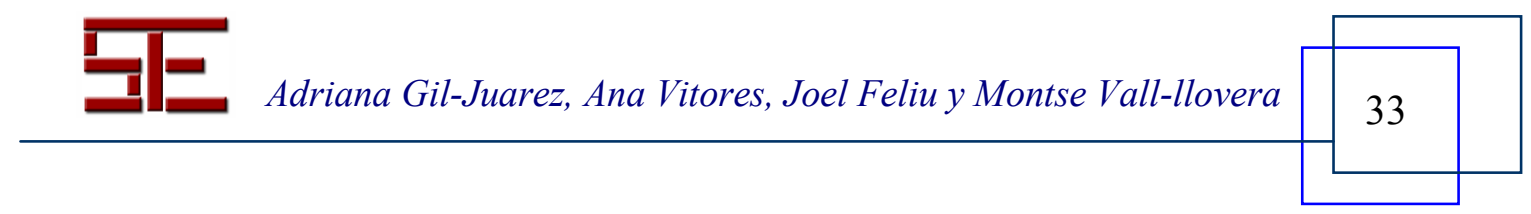




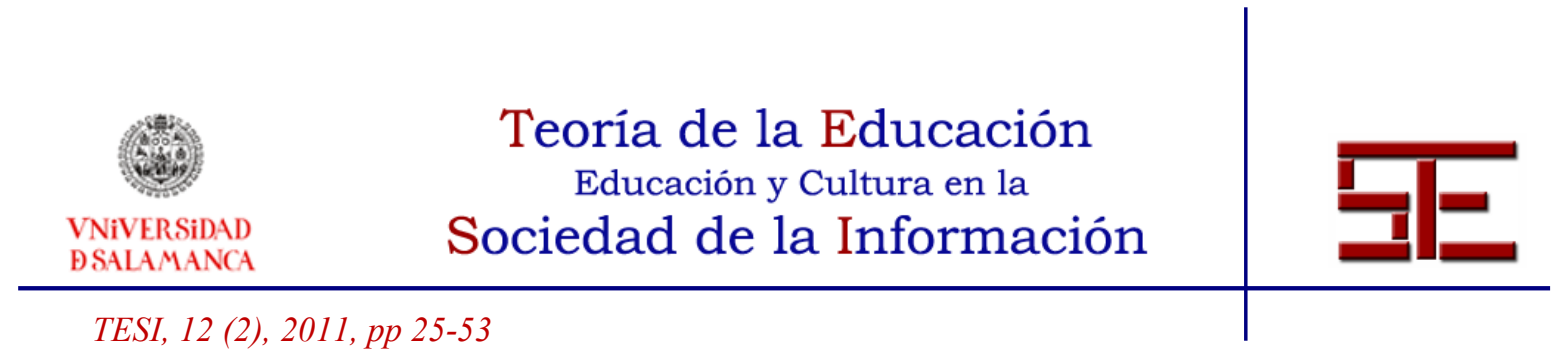

interesadas por ellos, incluso muestran más ansiedad frente a ellos (computer anxiety) (He y Freeman, 2009; Meelissen y Drent, 2008; Todman, 2000). Una desafección o ansiedad hacia los ordenadores que se explica como fruto de una menor confianza en las propias habilidades con ellos (Margolis \& Fisher, 2002). En efecto, los estudios señalan que las chicas sistemáticamente infravaloran sus habilidades tecnológicas respecto a las que realmente son y que muestran mucha menor confianza que los hombres en sus habilidades en este terreno (Meelissen and Drent, 2008; Vekiri y Chronaki, 2008; Papastergiou, 2008; Van Braak, 2004).

Tanto para imprimir afección por las TIC como comodidad y confianza con ellas, se encuentran en la literatura dos aspectos que se consideran centrales: por un lado, el tener experiencias positivas con ellas desde la infancia y la juventud y, por otro lado, que el aprendizaje de las tecnologías sea informal. Respecto a las experiencias positivas tempranas, los estudios muestran que las chicas no tienen experiencias con las TIC tan positivas como los chicos: su relación con ellas deviene problemática en la adolescencia, aunque se origina antes (Baldassarri, 2009; Hackbarth, 2001). Así, por ejemplo, ya hacia los 11-12 años las chicas empiezan a expresar aburrimiento e incluso rechazo y aversión hacia la informática y hacia la dimensión "técnica" de los ordenadores (Anderson et al., 2008); unas actitudes negativas que se pronuncian todavía más a partir de los 15-16 años (Colley y Comber, 2003). De este modo, las varias relaciones que chicas y chicos van estableciendo con las TIC contribuyen a la conformación de identidades como usuarios/as de TIC diferentes, con aptitudes y sentimientos de competencia con las TIC diferenciados, $\mathrm{y}$, finalmente, intereses desiguales en profesiones vinculadas a ellas (Margolis y Fisher, 2002).

Asimismo, en la adquisición de esa relación positiva con las TIC juegan un papel central la experiencia y al aprendizaje informal. Incluso cuando se tiene acceso a un aprendizaje formal en tecnologías informáticas, el factor clave en la adquisición de competencias, afección, confianza e interés en las TIC es el aprendizaje en contextos informales (Faulkner y Lie, 2007). Son varios los estudios que muestran que aunque no se dan diferencias significativas de género en el tiempo y los usos que los chicos hacen de los ordenadores en el contexto escolar, sí se dan fuera de él. Así, por ejemplo, mientras que las chicas reportan ante todo usos del ordenador e Internet para fines comunicacionales o para el trabajo escolar, los chicos reportan más usos expertos en informática, como la creación de páginas web o la programación (Broos \& Keith, 2006; Hakkarainen, 2001; Volman et al., 2005). En especial, los chicos reportan mayor uso de los ordenadores con fines lúdicos (Hakkarainen et al., 2000; Mumtanz, 2000), y más

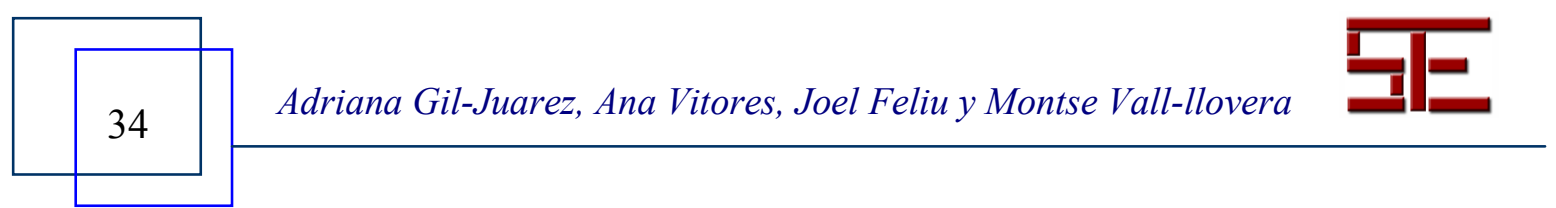




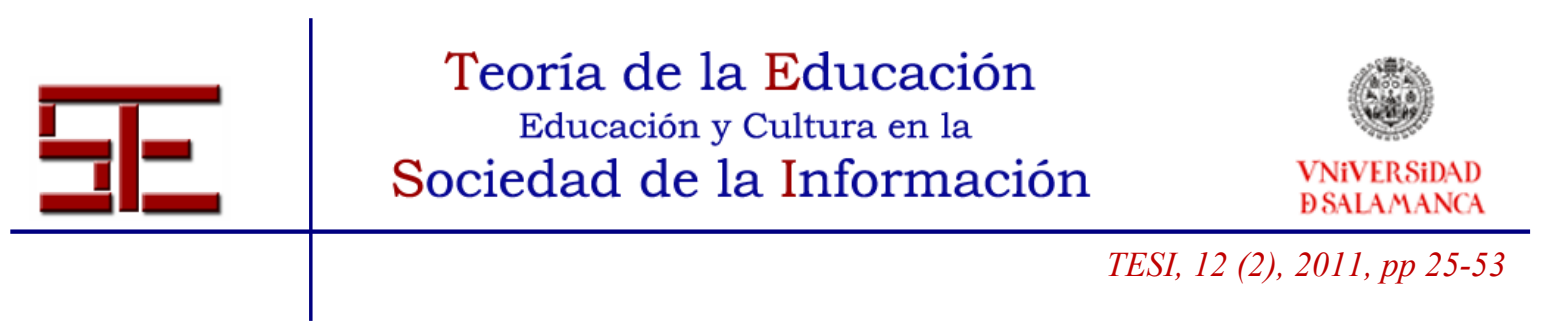

concretamente para jugar a videojuegos (Gil y Vall-llovera, 2006, 2009; Kent y Facer, 2004, McCoy y Heafner, 2004).

\section{1.- Socialización de género y TIC}

Aunque, como hemos comentado, el porcentaje de mujeres estudiando y trabajando en las TIC sea muy reducido en la gran mayoría de países occidentales, no estamos ante una tendencia universal ni constante. En efecto, los estudios realizados en algunos países no occidentales muestran porcentajes más elevados de mujeres "en las TIC" (Kelkar et al., 2005; Lagensen, 2008; Saloma-Akpedonu, 2005; Wacjman \& Lobb, 2007). Así, por ejemplo, en países como Taiwán (Fan \& Li, 2004) o Mauricio (Adams et al., 2003) las mujeres entran y se gradúan en estudios de informática en porcentajes similares al de los hombres y en Filipinas el 30\% de las personas que componen la Philippine Computer Society son mujeres (Saloma-Akpedonu; 2005; Wajcman \& Lobb, 2007). Del mismo modo, si atendemos al detalle de los contextos específicos en los países occidentales, encontramos estudios como el de Antonio López y Lisa Schultze (2002) que muestra cómo en la brecha entre hombres y mujeres que se licencian en Informática en Estados Unidos se halla escondida una excepción: en las universidades y escuelas universitarias estadounidenses históricamente afroamericanas (HBCUs) no se da tal brecha. En este sentido, quizás uno de los estudios más interesantes sea el de Ulf Mellström (2009), sobre la situación en Malasia, un país en el cual la informática es cosa de mujeres. El caso de Malasia es muy informativo, dado que es la discriminación de género de la sociedad tradicional malaya, apoyada sin querer por una política restrictiva de acceso a la educación superior para "no malayos", la que lleva a las mujeres a la informática, dado que se considera que un trabajo en espacios cerrados como el desarrollo de software es más conveniente para ellas.

Este tipo de apreciaciones son una buena prueba de que no son las supuestas características intrínsecas de las mujeres las que las llevan a no interesarse por las TIC y que, por lo tanto, debemos buscar el origen del problema en el contexto socio-cultural. Seguramente, el argumento definitivo nos lo proporciona la mirada histórica al caso de Estados Unidos: cuando la informática se estaba aún conformando como disciplina a mediados del siglo XX, el porcentaje de mujeres era relativamente alto. En efecto, en la década de 1950, la generación pionera de la informática incluía un número elevado de mujeres que lideraron equipos de trabajo y definieron lenguajes de programación (Light, 1999; Misa, 2010). La cumbre se da en 1980: a mediados de esa década las mujeres obtuvieron el 30\% de las licenciaturas en informática de Estados Unidos (Sanders,

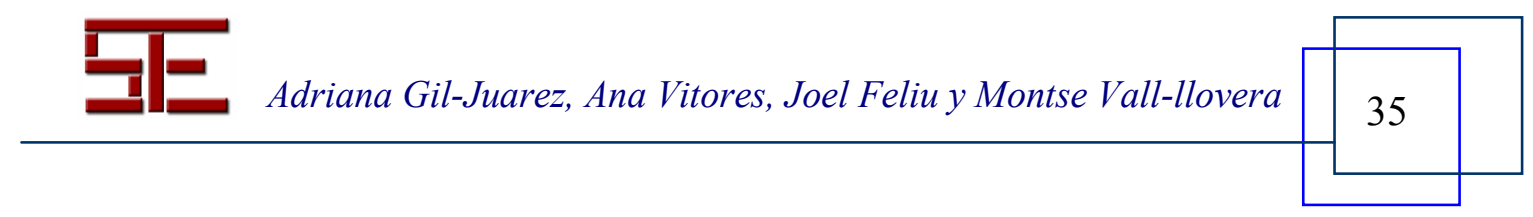




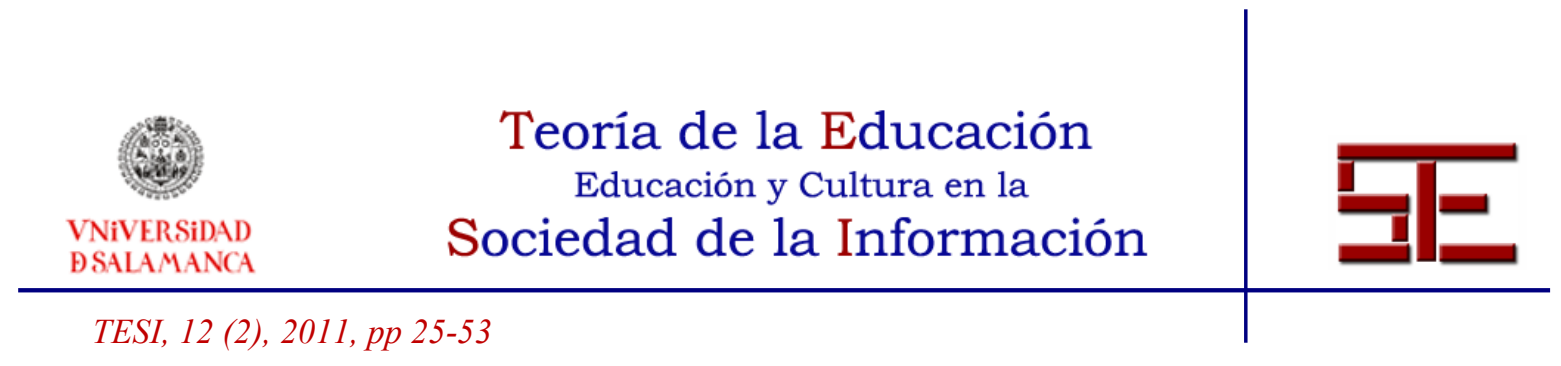

2006), de acuerdo con los datos que ofrece Jo Sanders (2006) en 1982 había más licenciadas en informática que en ingeniería, física o química. A finales de esa década las mujeres constituían el 38\% del personal de "cuello blanco" del sector informático, habiendo proporcionalmente más mujeres trabajando en informática que en cualquier otro campo tecnológico (Misa, 2010).

De este modo, el grueso de los estudios en este campo apunta a que para comprender mejor la problemática relación que se da entre las mujeres y las TIC debemos buscar las causas en los condicionantes sociales y educativos vinculados a la socialización de género (Jenson y De Castell, 2010; Misa, 2010). A través de la socialización diferencial de género, hombres y mujeres interiorizamos normas, valores y formas de hacer y decir adecuadas a nuestro sexo en función de la sociedad en la que nacemos. Y, entre éstas, aprendemos normas, valores y formas diferenciadas de relacionarnos relativas al ámbito tecnológico. En este sentido, la falta de interés hacia las tecnologías por parte de las mujeres estaría vinculada a la construcción sociocultural de la tecnología como un ámbito y una actividad adecuada para los hombres (Wajcman, 2006). Para conocer cómo se construye y naturaliza esta relación diferencial con las tecnologías en función del género, es necesario atender al papel de las tecnologías en la conformación de las relaciones de género presentes en una sociedad, para comprender, por ejemplo, cómo la masculinidad se construye en parte a través de asunciones relativas a determinadas competencias y habilidades tecnológicas y la feminidad a través de asunciones sobre la ausencia de esas mismas habilidades (Grint ; Gill, 1995).

Aunque la competencia tecnológica no se defina únicamente en relación el género, sí que tiene una relación estrecha con éste: se considera a los hombres como competentes con las tecnologías en virtud de su masculinidad, y las mujeres se muestran como carentes de esas mismas competencias cuando se comportan "como tales". Incluso las mujeres que usan y se muestran competentes con determinadas tecnologías son descritas como menos femeninas o incluso andróginas (Royse et al., 2007), lo que muestra hasta qué punto la competencia tecnológica es un acto interpretado como expresivo de un núcleo de identidad de género masculina. Ése es el argumento que esgrimen autoras como Cynthia Cockburn (1992) o Judy Wajcman $(1991,2006)$ para explicar por qué las mujeres muestran reactancia o resistencia a participar en tecnologías masculinizadas. El ordenador, como una de esas tecnologías claramente masculinas, supone un cuestionamiento de su identidad como fémina, ya que forma parte de prácticas claramente categorizadas como actividades de hombres. O, como concluye Sherry Turkle (1988), cuando las mujeres expresan una falta de interés en los ordenadores, no

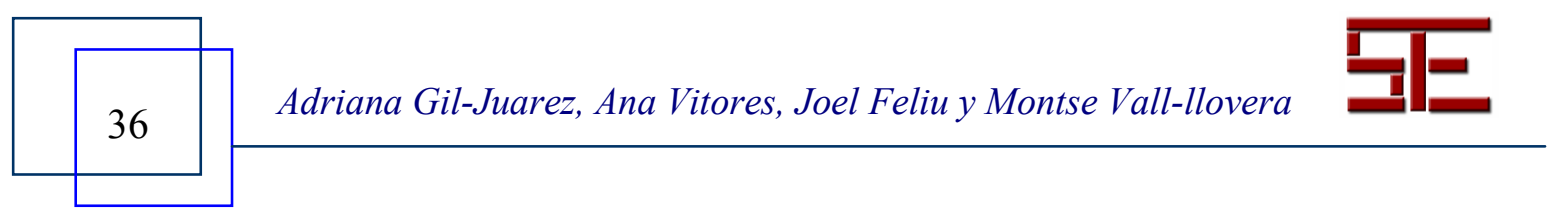




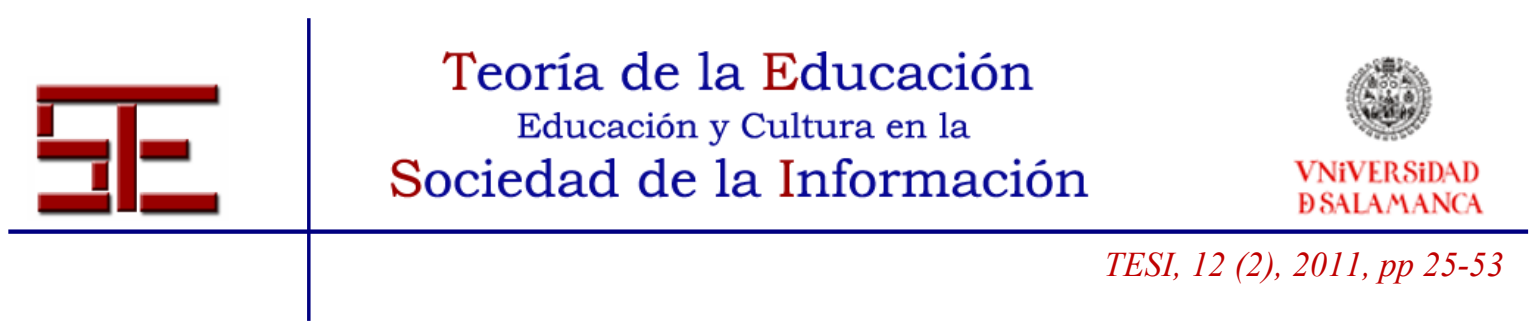

muestran una fobia sino más bien una reticencia: están diciendo algo sobre quiénes son ellas mismas.

El papel de la socialización de género en la conformación de la competencia y la afección hacia las tecnologías se observa de forma elocuente en el ejemplo de la brecha digital de género que existe en los videojuegos, una brecha que múltiples trabajos vinculan con una disminución de las oportunidades educacionales y profesionales para las mujeres (Beavis y Claire, 2007; Hayes, 2008; Jenson y De Castell, 2005; Kafai, 1998; Subrahmanyam y Greenfield, 1998). En efecto, los videojuegos por su carga lúdica, que hace que su consumo sea facultativo e informal, se han convertido en un foco de investigación privilegiado para el estudio de los mecanismos que mantienen a las chicas alejadas del mundo tecnológico, sin tener que apelar a razones o mecanismos institucionales, que no explican correctamente las exclusiones "voluntarias", es decir, aquellas que se producen porque la persona "no quiere" o "no está interesada" en participar en el mundo de las TIC. En nuestros propios trabajos (Gil y Vall-llovera, 2009; Gil, Feliu y Vitores, 2010) hemos detectado una creciente desafección por el ocio tecnológico en las chicas a medida que se hacen mayores. Aunque las causas de esta desafección son diversas y complejas, uno de los aspectos más sobresalientes es la conflictiva situación que se crea para las chicas cuando se encuentran en el "dilema" de ejecutar un rol femenino y ejecutar un rol de videojugadora. Como Valerie Walkerdine (2006) ya apuntó, las niñas se encuentran en un entorno difícil al jugar a videojuegos, tratando de performar la feminidad que se les adscribe y al mismo tiempo la masculinidad adscrita a los videojuegos. Así, por ejemplo, si quieren parecer competitivas, como hay que serlo en los videojuegos, entonces tienen que dejar de lado la cooperación, pero eso parece contradecir un mandato tradicional del rol femenino.

En el aprendizaje y la ejecución de este rol femenino juegan un papel importante las expectativas de los padres y las madres como modelos y referentes. El tipo de relación que las madres desarrollan con las tecnologías se convierte en el modelo femenino de identificación de las niñas. A la vez, las visiones de padres y madres sobre los videojuegos como cosas de "niños", así como las expectativas sobre el desarrollo y maduración de las niñas que tienen (que se asume maduran antes $\mathrm{y}$, por lo tanto, pierden el interés por jugar antes que los niños) refuerzan que se considere la relación entre las niñas y los videojuegos como algo problemático, y la relación entre niños y videojuegos como un fenómeno "natural” (Gil-Juárez et al., en preparación; Vitores, et al., 2010).

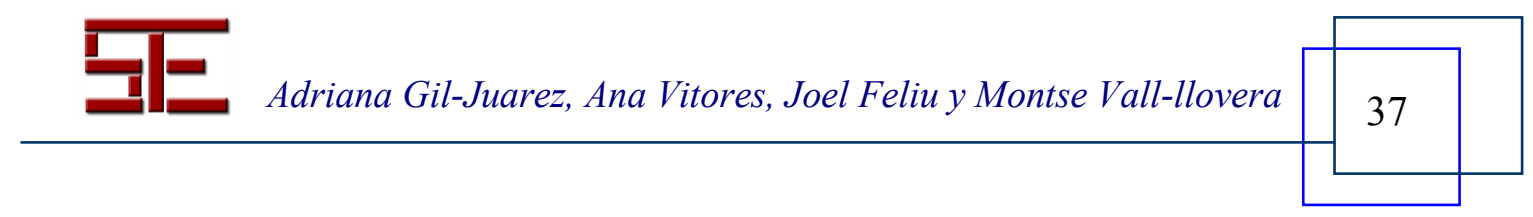




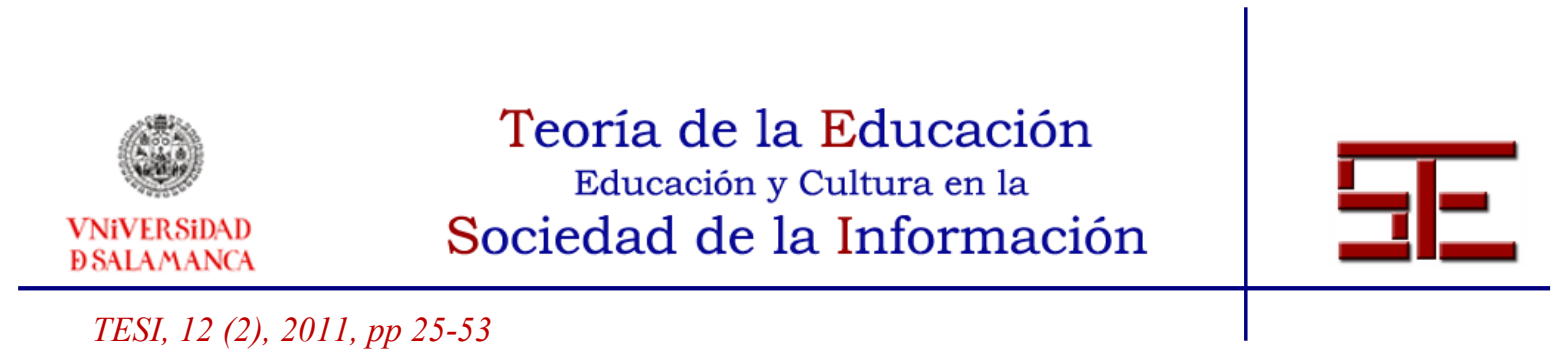

Considerar la socialización de género como la principal "barrera" que dificulta el interés y el enrolamiento de las mujeres en las TIC, es afortunadamente distinto a achacarlo a una falta de disposición inherente o una "carencia" en sus competencias. Sin embargo, a nivel práctico, aunque muchos de los estudios y las intervenciones asumen teóricamente la socialización de género como "el problema", paradójicamente promueven una lectura en términos de carencias de las mujeres. En otras palabras, son las niñas y las mujeres principalmente las destinatarias de los estudios y las intervenciones, son ellas, en definitiva, las que se presentan como deficitarias en relación a las tecnologías. Así, señalan autoras como Jane Abiss (2008), no sólo es la profesión en las TIC la que está discursivamente situada en términos de norma masculina y déficit femenino, sino que también lo está buena parte de la investigación sobre género y TIC que marca a las mujeres como "carentes de" o "resistentes a": "La inclinación a culpar a las mujeres por su falta de implicación en la industria de las TIC y en cursos de informática "dura" es en sí misma un reflejo de la estructuración social del género y de las TIC" (Abiss, 2008, 162). A nuestro entender, y como expondremos a continuación, en buena medida esto responde al error de traducir "socialización de género" en "socialización de las mujeres" (Adam et al., 2004).

\section{3.- EL GÉNERO EN/Y LA TECNOLOGÍA}

Como hemos comentado, el enfoque y el objetivo actual de muchas propuestas se basan en situar el principal indicador del alcance del problema de la brecha digital de género en la inferioridad numérica de las mujeres EN las TIC. Consecuentemente las intervenciones se dirigen a aumentar el número de mujeres que acceden a estudios y trabajos en ese ámbito, tratando de hacer más accesibles o atractivas las tecnologías a las mujeres. Y aunque, en efecto, sea importante aumentar el número de mujeres que acceden y entran en este ámbito la presencia minoritaria de las mujeres en las TIC no sólo es, quizás ni principalmente, una cuestión numérica.

Si se postula que una de las medidas más cruciales para atraer a las mujeres a los estudios y trabajos en las TIC es hacer un esfuerzo directo por incrementar el número de mujeres en estos entornos (Lagensen, 2007) es porque se entiende que aumentar la presencia de mujeres en este ámbito incide en la percepción simbólica de las TIC de ser algo también "para mujeres". Así, el pivote de la pregunta y la intervención no debería descuidar que no es el "número" en sí el problema a enfrentar, sino el simbolismo masculino de las TIC. Recordar esto nos permitirá no reducir la pregunta de investigación a por qué las mujeres no están en las TIC hoy, sino indagar también en cómo el ámbito de las TIC se ha ido conformando como un entorno masculino

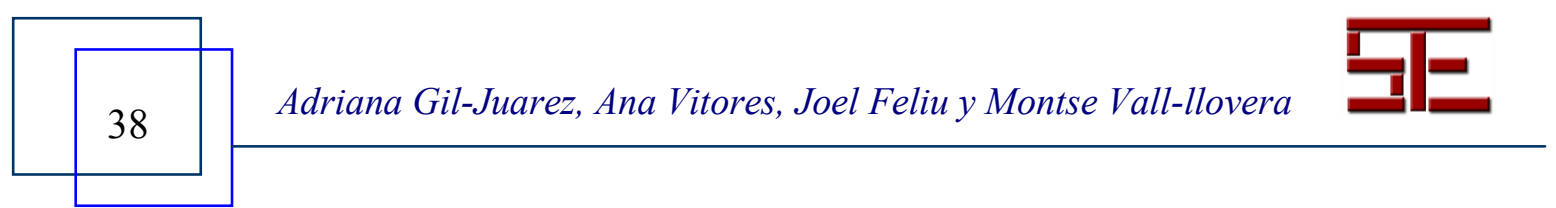




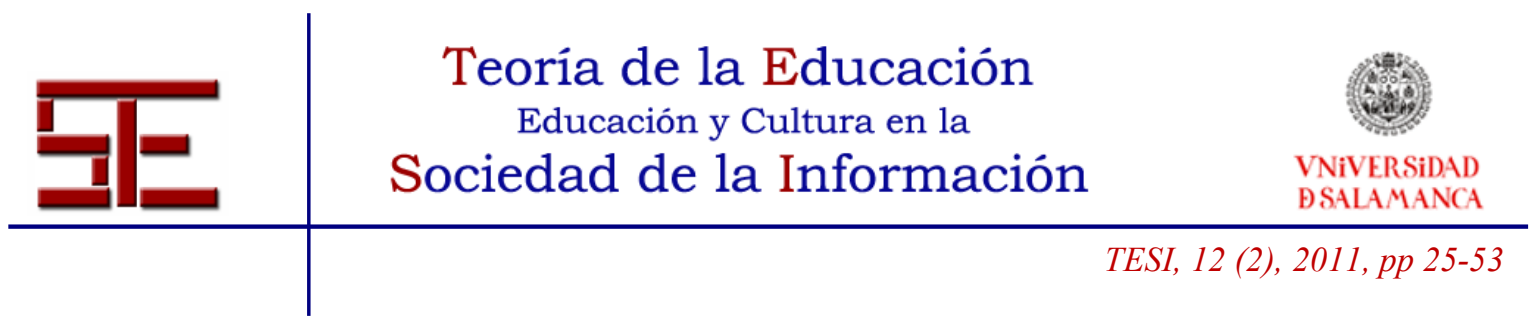

(Corneliussen, 2009; Misa, 2010) y, consecuentemente, cómo podemos revertirlo para evitarnos tener que eternizar unas poco eficaces campañas para aumentar el número de mujeres en carreras tecnológicas.

Asimismo, en las propuestas para aumentar el acceso de mujeres a estas carreras existe un énfasis en incidir en las barreras que lo impiden, ya que, como hemos señalado, la mayor parte de la investigación y la intervención está centrada en aquellos factores que excluyen a las mujeres del mundo TIC. Sin embargo, se sabe poco de los factores que inciden en su inclusión: aunque sea en bajos porcentajes, sí hay, en nuestro contexto cultural, mujeres que disfrutan con las tecnologías, desarrollan un uso experto de las mismas y optan por estudiar y trabajar en este ámbito. Sin embargo, aunque todavía son escasos los estudios que aportan conocimientos sobre las mujeres incluidas en las TIC, la mayoría de ellos son estudios que visibilizan ante todo las dificultades que encuentran las mujeres al estudiar y trabajar en estos entornos (Faulkner, 2007, 2009; Webster, 2005) así como los factores que inciden en su abandono (Adam et al., 2006; Griffiths y Moore, 2010). Podríamos decir que de igual modo que las mujeres en el sector TIC y las carreras informáticas son poco visibles en nuestra la sociedad, sus problemas son demasiado visibles entre quienes estudian las mujeres en las TIC (Boivie, 2010; Mellström, 2010). Es decir, que estos estudios se centran, a su vez, en los problemas que tienen estas mujeres, antes que en lo que las llevó donde están, y lo que ayuda a mantenerlas ahí. En este sentido, es necesario conocer mejor aquellos factores que inciden positivamente en la atracción hacia entornos TIC, así como en su inclusión efectiva y satisfactoria en esos entornos. Aunque a menudo se entiende la inclusión como la eliminación de los factores excluyentes, debemos abordarla como un proceso en el que la atracción y afección hacia las TIC es tan importante como la ausencia de desafección hacia ellas (Faulkner y Lie, 2007).

El efecto del énfasis en los números y en las barreras en este ámbito de estudio revela una lógica particular de este campo de investigación e intervención que a menudo confunde la urgencia con la importancia. Si bien hay urgencia, en nuestra sociedad, de incrementar el número de mujeres en las TIC y conseguir que el máximo número de personas se incorporen a los sectores vinculados a la tecnología, esto no debería impedirnos ver la importancia de entender, conocer e incidir en cómo se están configurando las relaciones entre género, tecnología y sociedad. Sin esta comprensión teórica no podremos desarrollar ningún instrumento de cambio social en la dirección que deseamos y necesitamos.

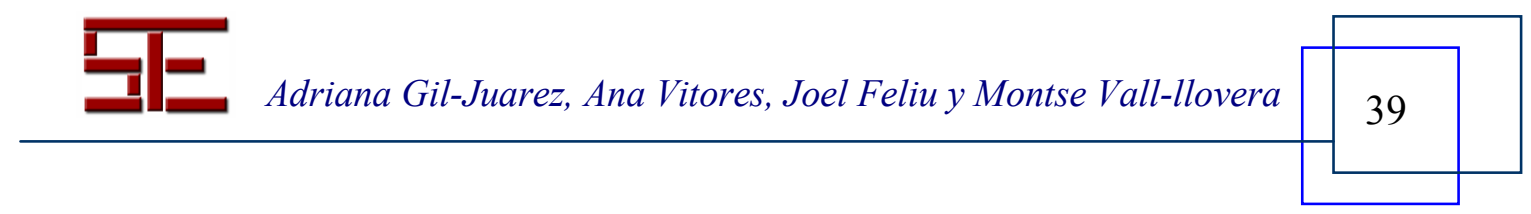




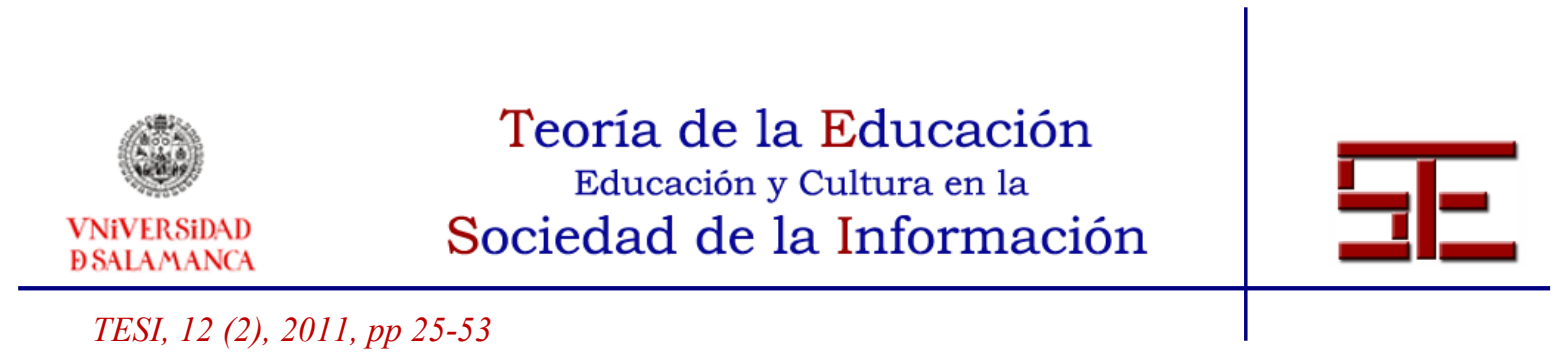

En este sentido, en las investigaciones sobre las mujeres en las TIC se detecta una falta de teorización sobre las relaciones entre género y tecnología (Adam et al., 2004; Singh et al., 2007). En efecto, aunque, como hemos comentado, son numerosos los estudios que describen usos y afectos diferenciales hacia las tecnologías en función del género, todavía queda mucho por conocer sobre cómo se construye y naturaliza esta relación diferencial (Faulkner, 2009; Mellstrom, 2010; Singh et al, 2007). Para avanzar en este sentido, los estudios de Ciencia, Tecnología y Sociedad (CTS) ofrecen una consolidada tradición teórica y metodológica que en las últimas décadas ha incluido el género de forma explícita en su agenda (Faulkner, 2001; Suchman, 2008; Wacjman, 2010). Aunque la perspectiva de género estuvo descuidada en la tradición de los estudios sociales de la ciencia y la tecnología, los estudios feministas constructivistas sobre género y tecnología han incorporado de forma efectiva esta mirada al estudio de las relaciones entre tecnología y sociedad (Cockburn, 1992; Wajcman, 1991). No ha sido ésta una incorporación a modo de complemento ni un simple añadido: desde el punto de vista de estas autoras, el género y la tecnología no son entidades separadas, sino algo mutuamente constitutivo y definitorio.

La apuesta implica por un lado, y como hemos apuntado, estudiar el papel de la tecnología en la conformación del género y las relaciones de género presentes en una sociedad y, entonces, de qué modo la masculinidad y la feminidad adquieren significado a través de las prácticas tecnológicas. Estos trabajos invitan a estudiar el papel de las relaciones del género en la conformación de las tecnologías que usamos: no sólo en cuanto a los usos de una tecnología, sino en el mismo proceso de diseño, desarrollo e implementación de dicha tecnología. Asimismo, estos planteamientos permiten abordar el género no sólo como variable ya dada para desagregar datos sobre usos o actitudes hacia la tecnología, sino como la construcción psicológica y socio-cultural de las características consideradas masculinas o femeninas, así como categoría práctica y relacional (West y Zimmerman, 1987). Como categoría práctica porque no se aborda la masculinidad/feminidad como categorías a las que nos adscribimos/adscriben de una vez y en un único momento de la socialización, sino que se aboga por examinar las actividades cotidianas a través de las cuales las personas reproducimos y representamos el género para entendernos a nosotros/as mismos/as y para entender a quienes nos rodean. Entre esas actividades a través de las cuales hacemos y representamos el género, son cada vez más centrales y ubicuas las actividades tecnológicas. Como categoría relacional, por no abogar por estudios de "hombres" y/o de "mujeres" que constaten usos o preferencias diferenciales para unos $\mathrm{y}$ otras, como si fueran grupos independientes. El género se construye tanto a partir de las relaciones internas, con el

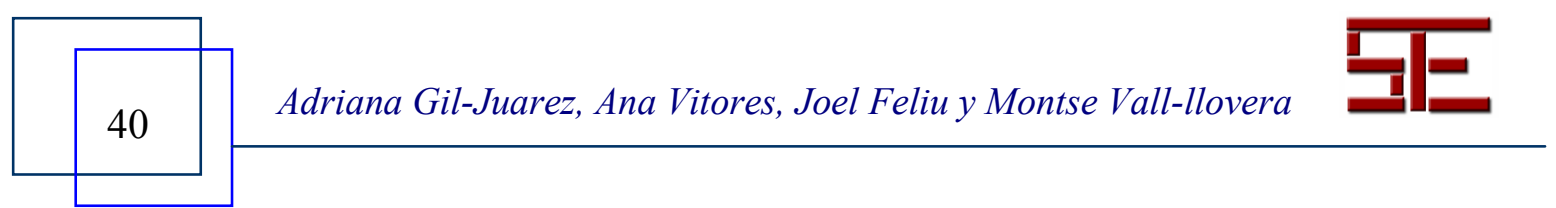




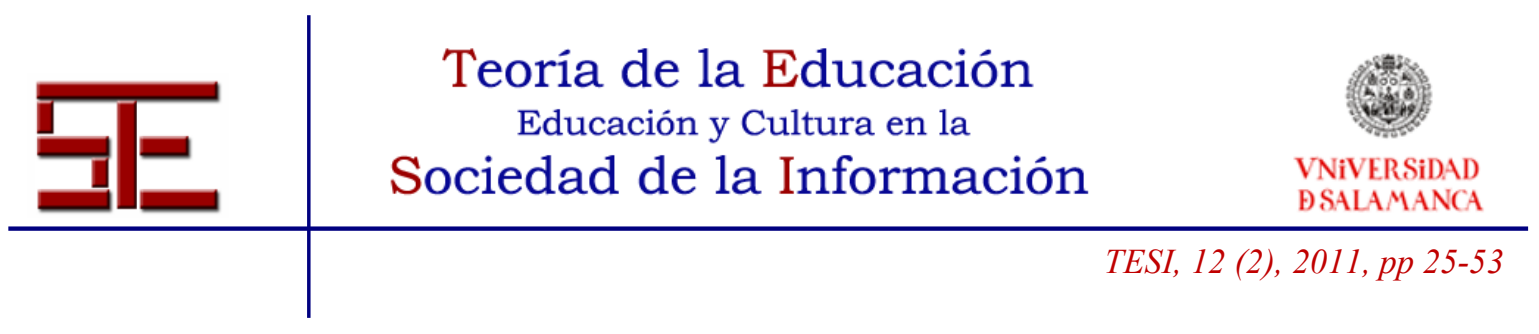

propio grupo, como con los miembros del otro grupo. La adscripción a un género no se realiza nunca de forma descontextualizada o aislada del otro género, sino siempre en presencia de un otro que representa lo que uno no es. Dicho de otra manera, las prácticas generizadoras tienen lugar también en relación al otro género y no solo al propio. Si para una niña las mujeres adultas de su entorno social y simbólico, familiares o no, pueden ejercer de modelo de lo que deberían ser, de lo que les debería gustar y de lo que deberían desear, los niños y los hombres que las rodean también ejercen su género de manera que ellas no se puedan identificar "erróneamente" con quien no deben parecerse. En este juego de identificaciones la relación con la tecnología es crucial puesto que a la tecnología se la define como más adecuada para un género que para otro, y en ese mismo gesto es tanto la tecnología como el género los que se construyen.

En este sentido creemos que es importante mantener una mirada poliédrica y resistente a la esencialización de los géneros y de la tecnología, que apueste por problematizar lo evidente y se pregunte por los procesos y prácticas concretas que hacen que género y tecnologías se conviertan en entidades obvias e incuestionables. En definitiva, se trata de no traducir ni reducir el problema de "las mujeres en las TIC" como el problema de las mujeres con las tecnologías. Es el género y la identidad femenina y masculina lo que está en cuestión. Entendemos esta identidad, como diría Butler (1998), como apariencias construidas mediante la reiteración de actos normativos que responden a interpelaciones concretas por parte de la sociedad en la que vivimos. Del mismo modo, y quizás éste es el mayor descuido, también es la tecnología la que está en cuestión. Si señalamos continuamente la distancia de las mujeres respecto a la tecnología, olvidamos preguntarnos por las tecnologías. Y no se trata de darle un barniz "rosa" a las tecnologías (Bautista y Martínez Tejeda, 2009). Se trata de preguntarnos por ellas y pensar en el porqué y cómo de su distancia respecto a una identidad de género concreta. Es decir, preguntarnos por la distancia de la tecnología, no de las mujeres, sino respecto a las posiciones minoritarias y minorizadas de nuestro sistema de género, y seguramente de otros sistemas.

En un momento en que las tecnologías de la información y la comunicación, convertidas en tecnologías de ocio y tecnologías de relación (Gil, 2007), permean cada una de las esferas de la vida, lo que está en juego no es solamente la cantidad de mujeres que tendremos creándolas y participando de ellas, sino el sistema de género que tenemos, y el que queremos para nuestra sociedad, si es que queremos alguno.

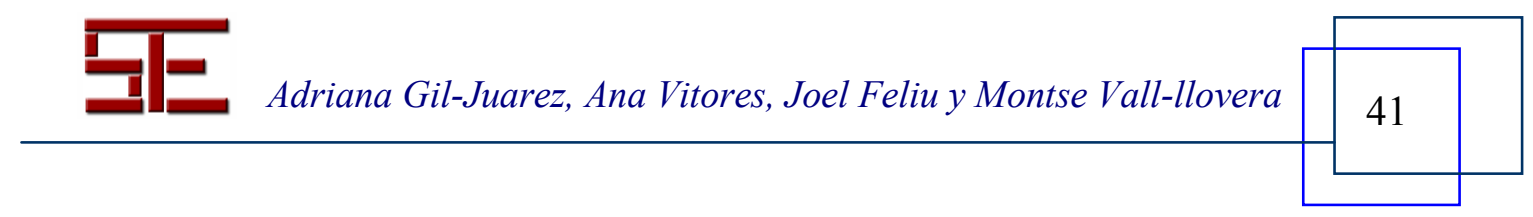




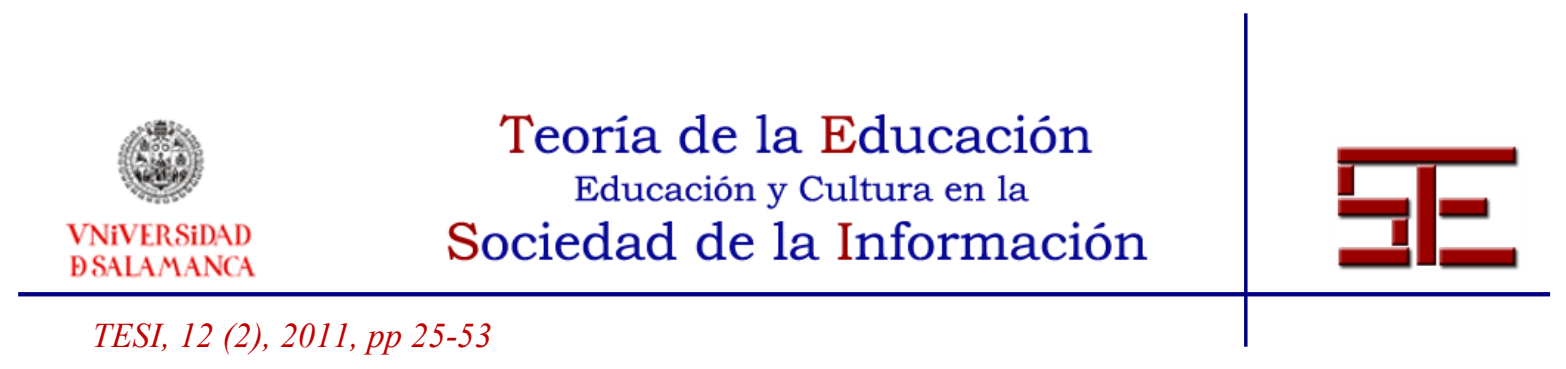

\section{4.- BIBLIOGRAFÍA}

AAUW (2000). Tech-Savvy: Educating Girls in the New Computer Age. Washington, DC: American Association of University Women Educational Foundation. Commission on Technology, Gender, and Teacher Education. Extraído el 13 de octubre, 2010, de http://www.aauw.org/learn/research/upload/TechSavvy.pdf .

Abbiss, J. (2008). Rethinking the "problem" of gender and IT schooling: Discourses in literature. Gender and Education, 20, 153-165.

Adam, A., Debra, H. \& Richardson, H. (2004). A decade of neglect: reflecting on gender and IS. New Technology, Work and Employment, 19 (3), 222-240.

Adams, J. C., Bauer, V. \& Baichoo, S. (2003). An expanding pipeline: gender in mauritius. SIGCSE Bull, 35 (1), 59-63.

Ahuja, M. K. (2002). Women in the information technology profession: a literature review, synthesis, and research agenda. European Journal of Information Systems, 11 (1), 20-34.

Anderson, N., Lankshear, C., Timms, C. \& Courtney, L. (2008). "Because it's boring, irrelevant and I don't like computers": Why high school girls avoid professionally-oriented ICT subjects. Computers \& Education, 50 (4), 13041318.

Ashcraft, C., Blithe, S. (2009). Women in IT: The Facts. Boulder, CO: National Center for Women \& Information Technology.

Baldassarri, S., Molina-Gaudo, P., Villarroya-Gaudo, M. \& Cerezo, E. (2009). The 1st Girls' Day at the University of Zaragoza (Spain). Extraído el 10 de enero, 2010 de http://www.unizar.es/mutem/pdfs/baldassarri_ICIE09.pdf .

Bautista, A. y Martínez Tejeda, G. (2009). Socialización y relacionalidad en el uso y apropiación de las TIC. En A. Gil y M. Vall-llovera (Coords.), Género, TIC y videojuegos. Barcelona: Editorial UOC.

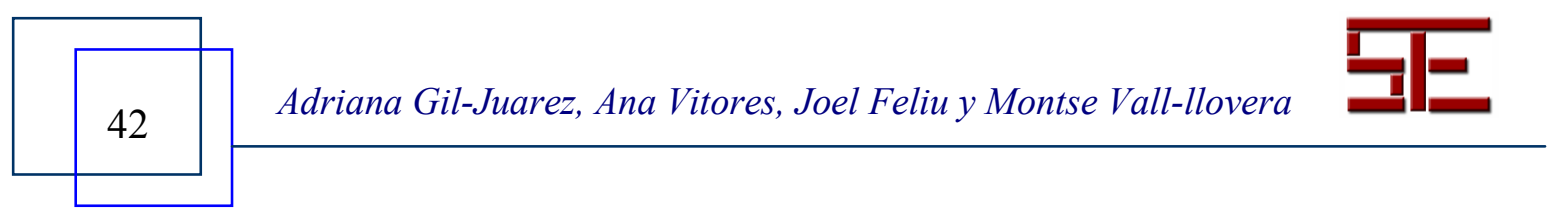




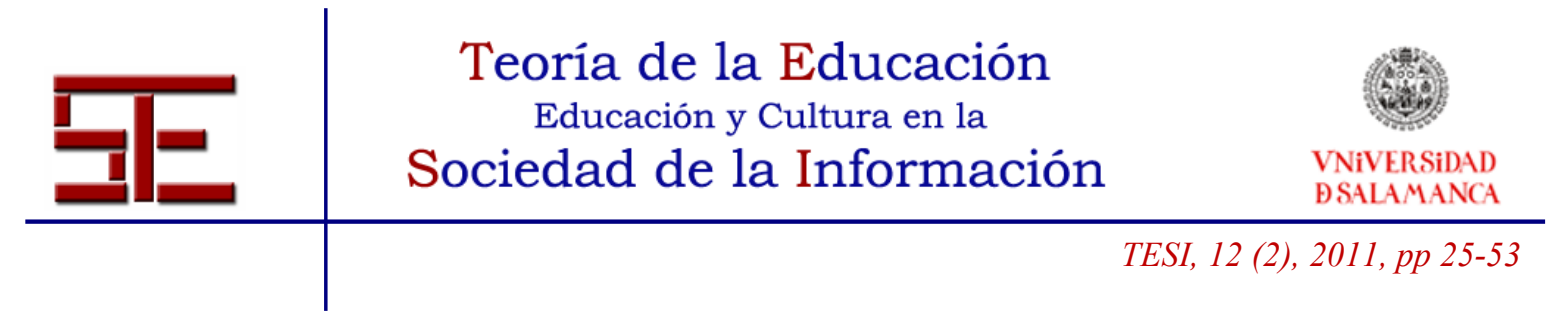

Beyer, S., Rynes, M., Chavez, M., Hay, K.; \& Perrault, J. (2002). Why are so few women in computer science? Comunicación presentada en el Annual Meeting of the American Psychological Association, New Orleans. Extraído el 14 de febrero, 2010 de http://www.eric.ed.gov/PDFS/ED479756.pdf.

BCS \& eSkills (2009). Women in IT Scorecard. The British Computer Society (BCS) y E-skills UK. Extraído el 14 de febrero, 2010, de http://www.ictwomendirectory.eu/digitalcity/servlet/PublishedFileServlet/AAA AWLPS/it-scorecard-bcs.pdf.

Blickenstaff, J. C. (2005). Women and science careers: Leaky pipeline or gender filter? Gender and Education, 17 (4), 369-386.

Black, S., Jameson, J., Komoss, R., Meehan, A. \& Numerico, T. (2005). Women in Computing: A European and International Perspective. Manchester, UK: Third European Gender and ICT Symposium: Working for Change. Extraído el 10 de enero, 2010, de http://ict.open.ac.uk/gender/2005/papers/black.word.

Boivie, Inger (2010). Women, Men and Programming. En S. Booth, S. Goodman \& G. Kirkup (Ed), Gender Differences in Learning and Working with Technology: Social Constructs and Cultural Contexts (p. 1-14). Hershey: IGI Global.

Booth, S., Goodman, S. \& Kirkup, G. (2010). Gender Differences in Learning and Working with Technology: Social Constructs and Cultural Contexts. Hershey: IGI Global.

Broos, A., Keith, R. (2006). The digital divide in the playstation generation: Self efficiancy, locus of control and ICT adoption among adolescents. POETICS, (34), 306-317.

Brynin, M. (2006). The Neutered Computer. En R. E. Kraut, M. Brynin \& S. Kiesler (Ed), Computers, phones, and the Internet (pp. 84-96). Oxford: Oxford University Press US.

Butler, J. (1998). Actos performativos y construcción del género. Debate Feminista, 18, 296-314.

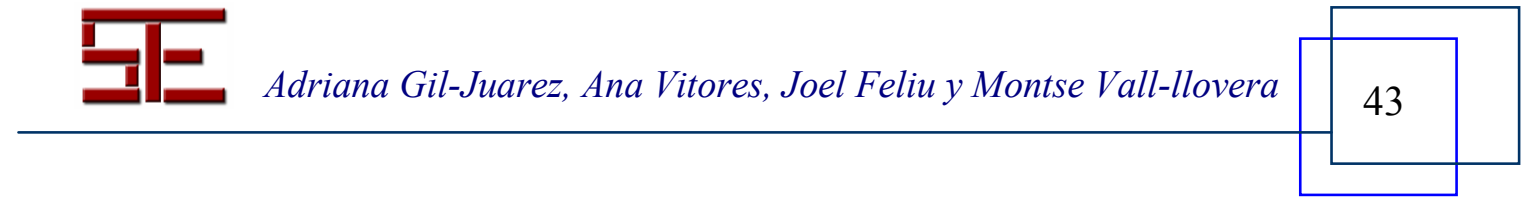




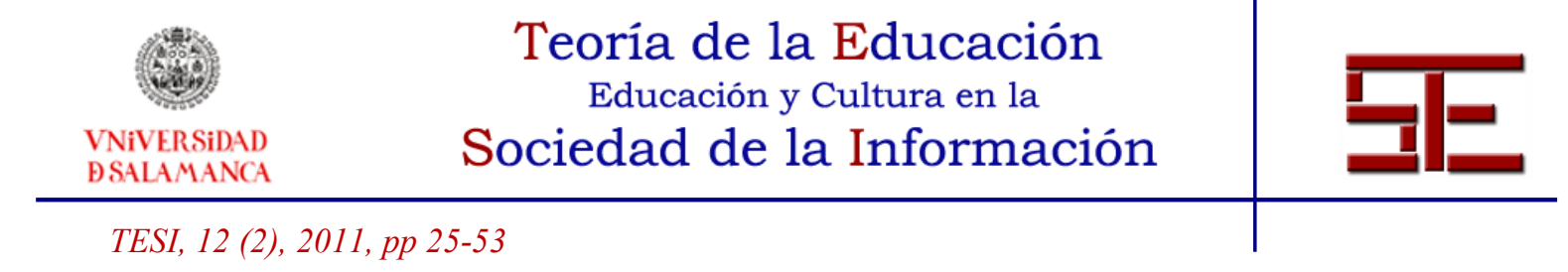

Camp, T. (1997). The incredible shrinking pipeline. Communications of the ACM, 40 (10), 103-110.

Castaño, C. (2008). La segunda brecha digital. Madrid: Ediciones Cátedra.

Castaño, C. (2009). La segunda brecha digital y las mujeres jóvenes. Quaderns de la Mediterrània, 11,218-224.

Castaño, C., Fernández, J. M., Vázquez, S. \& Martínez, J. L. (2009). La Brecha Digital de Género: Amantes y distantes. Madrid: Observatorio E-Igualdad de la Universidad Complutense de Madrid.

Clarke, C. (2010). Computer Science. The Incredible Shrinking Woman. En J. T. Misa (Ed). Gender Codes. Why Women Are Leaving Computing. Hoboken, New Jersey: Wiley-IEEE Computer Society Press.

Cockburn, C. (1992). The Circuit of Technology: Gender, Identity and Power. En R. Silverstone \& E. Hirsch (Eds.), Consuming Technology: Media and Information in Domestic Spaces (pp.32-47). London: Routledge.

Cohoon, J. M. \& Aspray, W. (2006). Women and Information Technology: Research on Underrepresentation. Cambridge: MIT Press.

Colley, A. \& Comber, C. (2003). Age and gender differences in computer use and attitudes among secondary school students: what has changed?. Educational Research, 45 (2), 155-165.

Córdoba, J. \& Jofre, L1. (2009). Anàlisi de la demanda d'estudis d'enginyeria. ENGINYCAT. Programa d'impuls a la innovació tecnològica des de la formació científica i tècnica. Extraído el 14 de febrero, 2010, de http://www.upc.edu/aprendre/estudis/activitats-informacio-orientacio/darreresactivitats/091202-presentacio-2-de-desembre-de-2009-v2.ppt.

Craig, A. (2006). A historical perspective of Australian women in computing. En E. Trauth (Ed.), Encyclopedia of gender and information technology (pp. 752-758). Hershey, PA: Idea Group Reference.

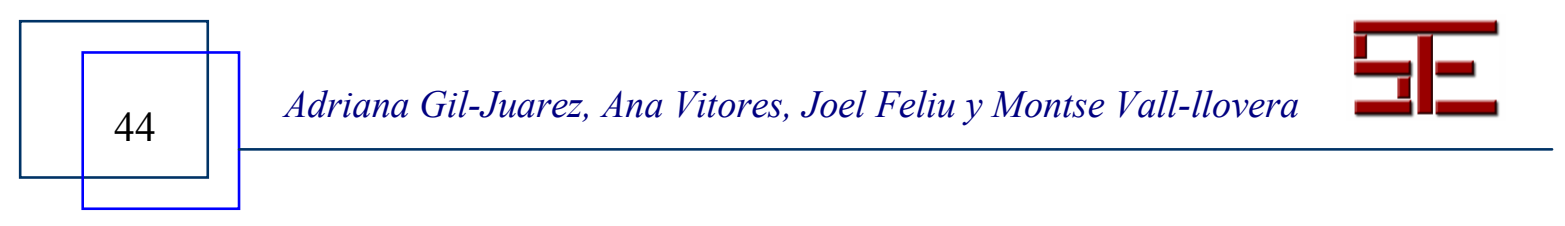




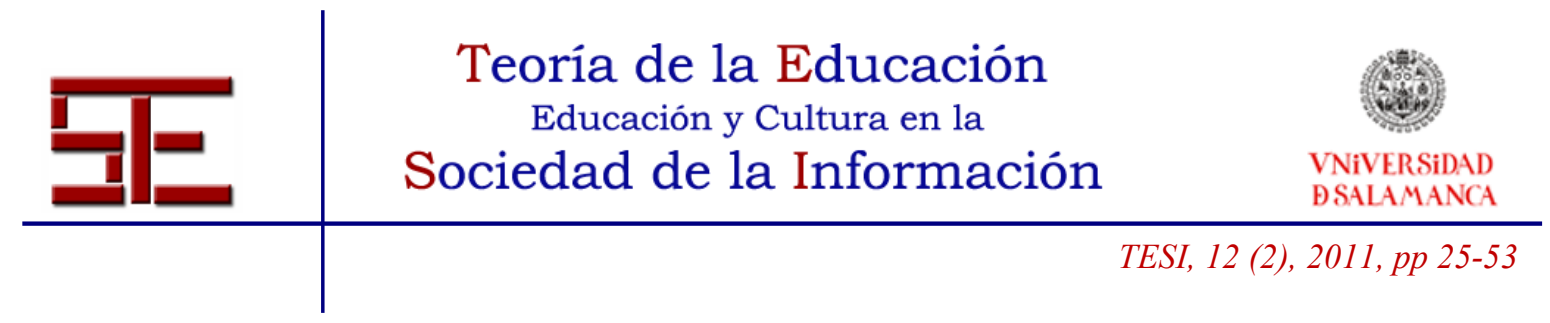

Cuny, J. \& Aspray, W. (2000). Recruitment and retention of women graduate students in computer science and engineering. Washington, DC: Computing Research Association.

Chinn, D. \& VanDeGrift, T. (2008). Gender and diversity in hiring software professionals: what do students say? Proceeding of the Fourth international Workshop on Computing Education Research (ICER '08). ACM, New York, NY, USA. Extraído el 20 de octubre, 2010, de http://portal.acm.org/citation.cfm?id=1404525.

Corneliussen, H.G. (2009). Disrupting the Impression of Stability in the GenderTechnology Relation. Ponencia presentada en el 5th European Symposium on Gender \& ICT, March 2009, University of Bremen, Germany. Extraído el 15 de marzo, 2010, de http://www.informatik.unibremen.de/soteg/gict2009/proceedings/GICT2009_Corneliussen.pdf.

De Palma, P. (2001). Viewpoint: Why women avoid computer science. Communications of the ACM, 44 (6), 27-30.

Eidelman, L. \& Hazzan, O. (2006). The shrinking pipeline in Israeli high schools. En E. M. Trauth (Ed.), Encyclopedia of gender and information technology (pp. 10921098). Hershey, PA: Idea Group Reference.

Eurostat (2009). Key data on education in Europe in 2009. Brussels: Education, Audiovisual and Cultural Executive Agency. Extraído el 20 de octubre, 2010, de http://epp.eurostat.ec.europa.eu/cache/ITY_OFFPUB/978-92-9201-0331/EN/978-92-9201-033-1-EN.PDF.

Fan, T.S., \& Li, Y.C. (2004). Gender issues and computers: College computer science education in Taiwan. Computers \& Education. 44, 285-300.

Fernández, V., Larraza, E., Ruiz, T. \& Maritxalar, M. (2008). Una aproximación a la situación de la mujer en los estudios universitarios de informática. Arbor, 184 (733), 877-887.

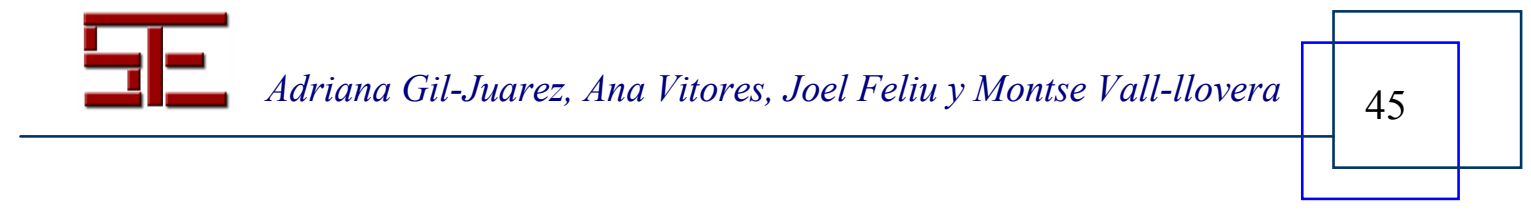




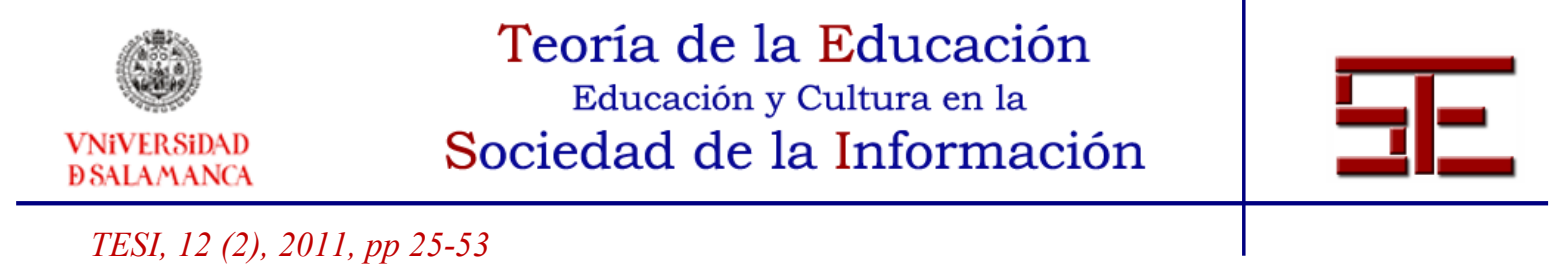

Faulkner, W. (2001). The technology question in feminism: a view from feminist technology studies. Women's Studies International Forum, 24,79-95.

- (2007). Nuts and Bolts and People: Gender-Troubled Engineering Identities. Social Studies of Science, 37, 331-356.

- (2009). Doing gender in engineering workplace cultures II. Gender in/authenticity and the in/visibility paradox. Engineering Studies, 1(3), 169-189.

Faulkner, W. \& Lie, M. (2007). Gender in the Information Society: Strategies of Inclusion. Gender, Technology and Development, 11 (2), 157-177.

Gal-Ezer, J.; Shahak, D. \& Zur, E. (2009). Computer science issues in high school: gender and more... SIGCSE Bulletin, 41(3), 278-282.

Gargallo-Castel, A., Esteban-Salvador, L. \& Pérez-Sanz, J. (2010). Impact of Gender in Adopting and Using ICTS in Spain. Journal of Technology Management \& Innovation, 5 (3), 120-128.

Gil, A. (2007). Nuevas Tecnologías de Relación. En J. Romay (Ed.), Perspectivas y Retrospectivas de la Psicología Social en los Albores del Siglo XXI. Madrid: Editorial Biblioteca Nueva.

Gil, A. \& Vall-1lovera, M. (2009). Género, TIC y videojuegos. Barcelona: Ediuoc.

Gil-Juárez, A., Feliu, J. \& Vitores, A. (2010). Performatividad Tecnológica de Género: Explorando la Brecha Digital en el mundo del Videojuego. Quaderns de Psicologia, 12 (2), 209-226.

Gil-Juárez, A., Feliu; J., Vitores, A. \& Vall-llovera, M. (artículo en preparación). Expressing and managing emotions in a gendered site: feelings, women and technology.

Glover, J. \& Guerrier, Y. (2010). Women in Professional IT Jobs in the UK: Old Wine in New Bottles? Journal of Technology Management \& Innovation, 5 (1), 85-94.

Gómez Ferri, J. (2004). La profesión de informátic@. Una investigación preliminar sobre la generización de la profesión. Comunicación presentada en el II

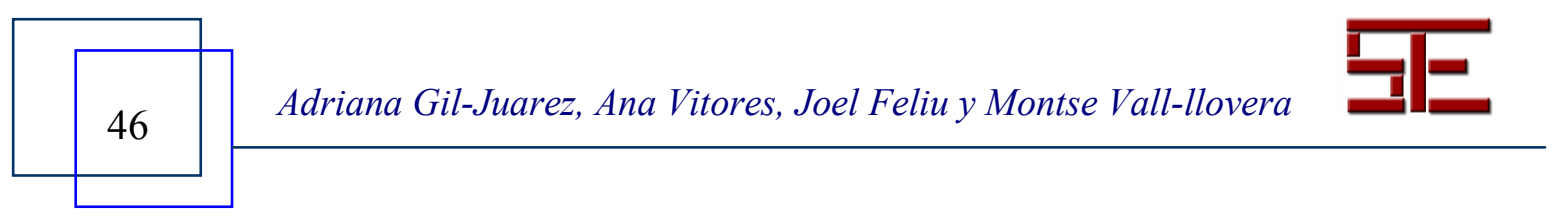




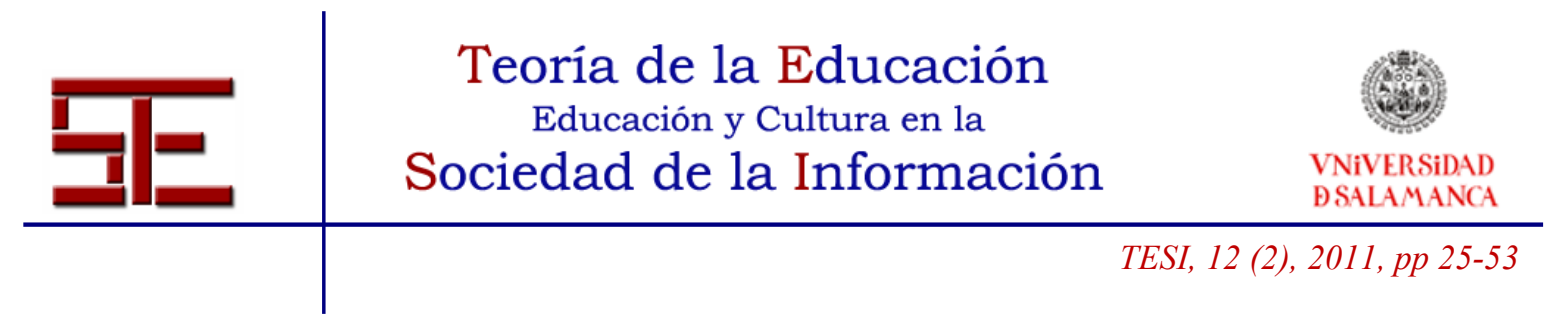

Congreso Online del Observatorio para la Cibersociedad. Extraído el 13 de febrero, 2011, de http://www.cibersociedad.net/congres2004/grups/fitxacom_publica2.php?idioma $=$ ca\&id $=533 \&$ grup $=48$.

Griffiths, M. \& Moore, K. (2010). Disappearing Women: A Study of Women Who Left the UK ICT Sector. Journal of Technology Management \& Innovation, 5 (1), 95107.

Grint, K. \& Rosalind, G. (1995). The Gender-Technology Relation: Contemporary Theory and Research. London: Taylor and Francis.

Gürer, D. \& Camp, T. (2002). An ACM-W Literature Review on Women in Computing. SIGSCE Bulletin, 34 (2), 121-127.

Hackbarth, S. L. (2001). Changes in Primary Students' Computer Literacy as a Function of Classroom Use and Gender. TechTrends, 45 (4),19-27.

Hakkarainen, K., Ilo“maki, L., Lipponen, L., Muukkonen, H., Rahikainen, M., Tuominen, T. et al., (2000). Students "e skills and practices of using ICT: Results of a national assessment in Finland. Computers and Education, 34 (2), 103-117.

Harris, N., Cushman, P., Kruck, S. E. \& Anderson, R. D. (2009). Technology Majors: Why Are Women Absent? Journal of Computer Information Systems, 50 (2), 23-30.

He, J. \& Freeman, L. (2009). Are Men More Technology-Oriented Than Women? The Role of Gender on the Development of General Computer Self-Efficacy of College Students. AMCIS 2009 Proceedings. Paper 672. Extraído el 18 de marzo, 2010, de http://aisel.aisnet.org/amcis2009/672.

Jenson, J. \& De Castell, S. (2010). Gender, Simulation, and Gaming: Research Review and Redirections. Simulation Gaming, 41, 51-71.

Kark, R. (2007). Women in the land of milk, honey and high technology: the Israeli case. En R. J. Burke \& M. C. Mattis (Eds.),Women and minorities in science, technology, engineering and mathematics: Upping the numbers (pp. 101-127). Cheltenham, UK: Edward Elgar Publishing Limited.

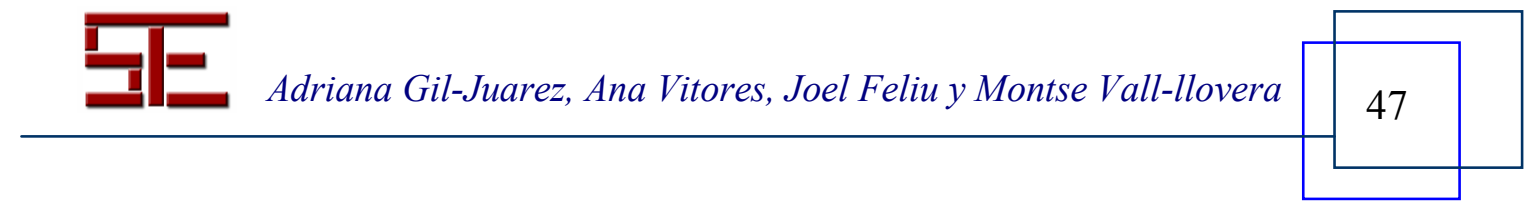




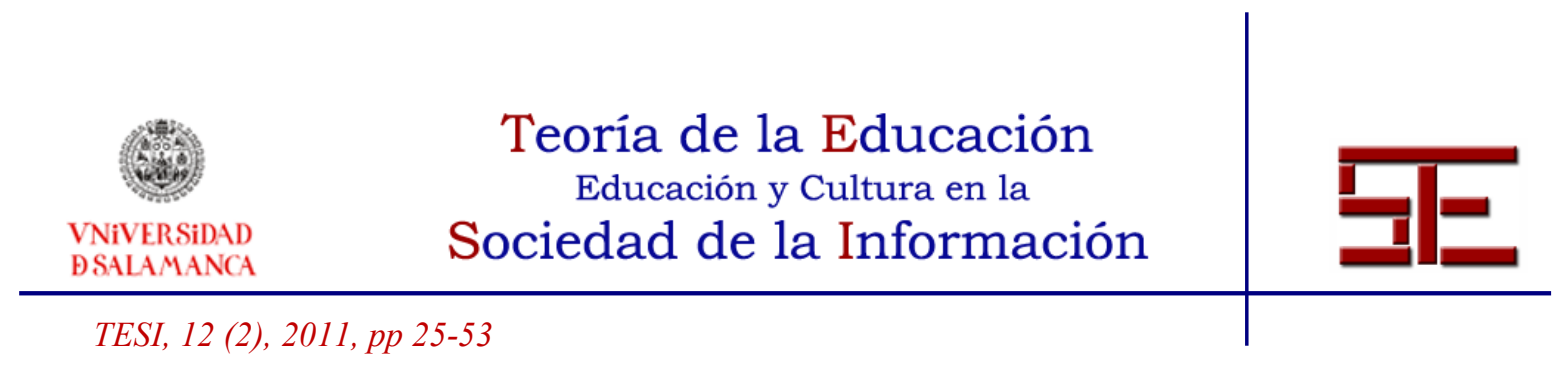

Kelkar, Govind, Shrestha, Girija and N. Veena. (2005) „Women“s Agency and the IT Industry in India. En Ng, Cecilia and Mitter, Swasti (Eds), Gender and the Digital Economy: Perspective from the Developing World (pp. 110-31). New Delhi: Sage Publications.

Kent, N. \& Facer, K. (2004). Different Worlds? Children's Home and School Computer Use. JCAL, 20, 440-455.

Klawe, M.; Whitney, T. \& Simard, C. (2009). Women in Computing-Take 2. Communications of the ACM, 52 (2), 68 -76.

Lagesen, V. A. (2007). The Strength of Numbers: Strategies to Include Women into Computer. Science Social Studies of Science, 37, 67-92.

- (2008). A Cyberfeminist Utopia?: Perceptions of Gender and Computer Science among Malaysian Women Computer Science Students and Faculty. Science, Technology \& Human Values, 33, 5-27.

Light, J. (1999). When Computers Were Women. Technology and Culture, 40 (3), $455-$ 483.

Lopez, A. M. \& Schulte, L. J. (2002). African American women in the computing sciences: a group to be studied. ACM SIGCSE Bulletin, 34 (1). Extraído el 14 de febrero, 2011,

de http://portal.acm.org/citation.cfm?id=563371\&CFID=23386071\&CFTOKEN=9 2174420 .

Margolis, J. \& Fisher, A. (2002). Unlocking the Clubhouse. Women in Computing. Cambridge: MIT Press.

McCoy, L. P. \& Heafner, T. L. (2004). Effect of gender on computer use and attitudes of college seniors. Journal of Women and Minorities in Science and Engineering, 10, 55-66.

McGrath Cohoon, J. (2001). Recruiting and retaining women in undergraduate computing major. Communications of the ACM, 44 (5), 108-114.

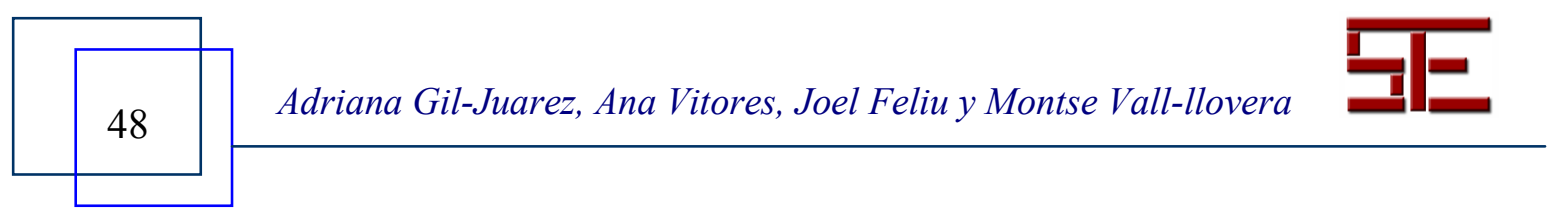




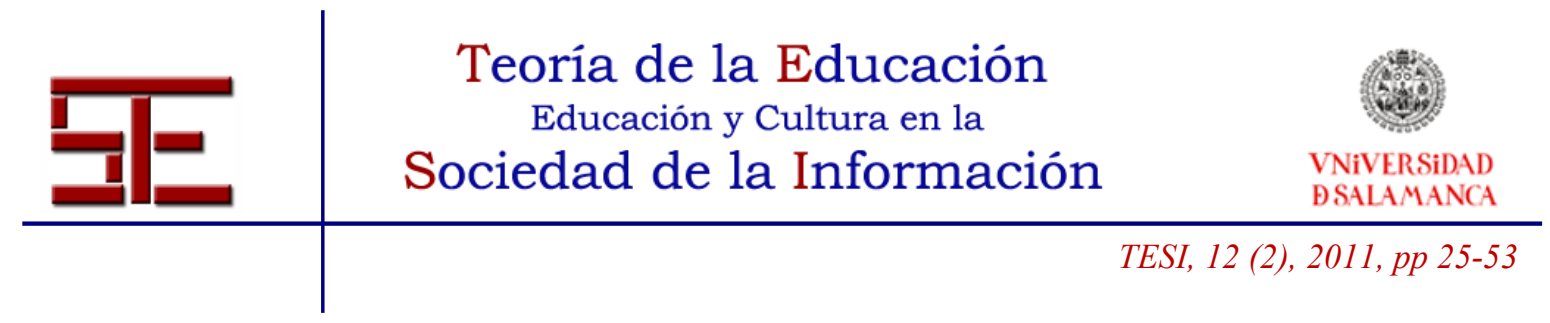

Meelissen, M. R. M. \& Drent, M. (2008). Gender differences in computer attitudes: Does the school matter? Computers in Human behavior, 24, 969-985.

Mellström, U. (2009). The Intersection of Gender, Race and Cultural Boundaries, or Why is Computer Science in Malaysia Dominated by Women? Social Studies of Science, 39, 885-907.

- (2010). New gender relations in thr Transforming IT-Industry of Malaysia. En S. Booth, S., Goodman \& G. Kirkup (Eds.), Gender Differences in Learning and Working with Technology: Social Constructs and Cultural Contexts. Hershey: IGI Global.

Metcalf, H. (2010). Stuck in the Pipeline: A Critical Review of STEM Workforce Literature. InterActions: UCLA Journal of Education and Information Studies, 6 (2). Extraído el 10 de febrero, 2011, de http://escholarship.org/uc/item/6zf09176

Miliszewska, I. \& Moore, A. (2010). Encouraging Girls to Consider a Career in ICT: A Review of Strategies. Journal of Information Technology Education, 9. Extraído el 15 de marzo, 2010, de http://jite.informingscience.org/documents/Vo19/JITEv9IIPp143166Miliszewska797.pdf .

Misa, T. J. (2010). Gender Codes: Women and Men in the Computing Professions. New Jersey: Wiley IEEE Computer Society.

Mumtaz, S. (2001). Children's enjoyment and perception of computer use in the home and the school. Computers and Education, 36(4), 347-362.

National Center for Women \& Information Technology (NCWIT) (2010). NCWIT Scorecard: A Report on the Status of Women in Information Technology. Extraído el 15 de marzo, 2010, de http://ncwit.org/pdf/NCWITScorecard2010_Workforce.ppt.

OCDE (2007). ICTs and Gender, DSTI/ICCP/IE. Extraído el 15 de marzo, 2010 de http://www.oecd.org/dataoecd/16/33/38332121.pdf.

Papastergiou, M. (2008). Are Computer Science and Information Technology still masculine fields? High school students' perceptions and career choices. Computers \& Education, 51 (2), 594-608.

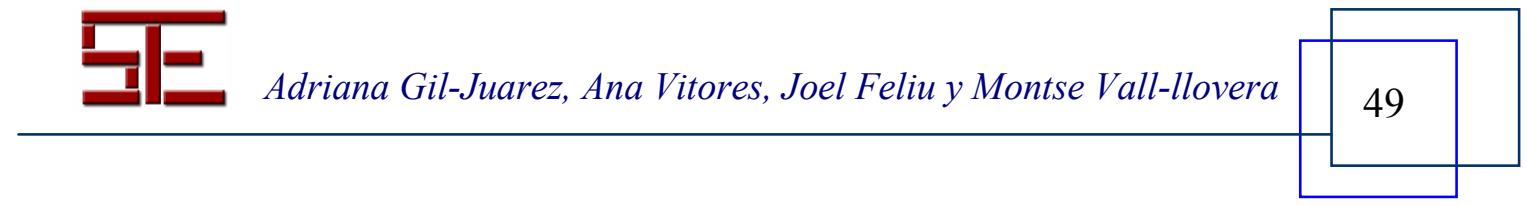




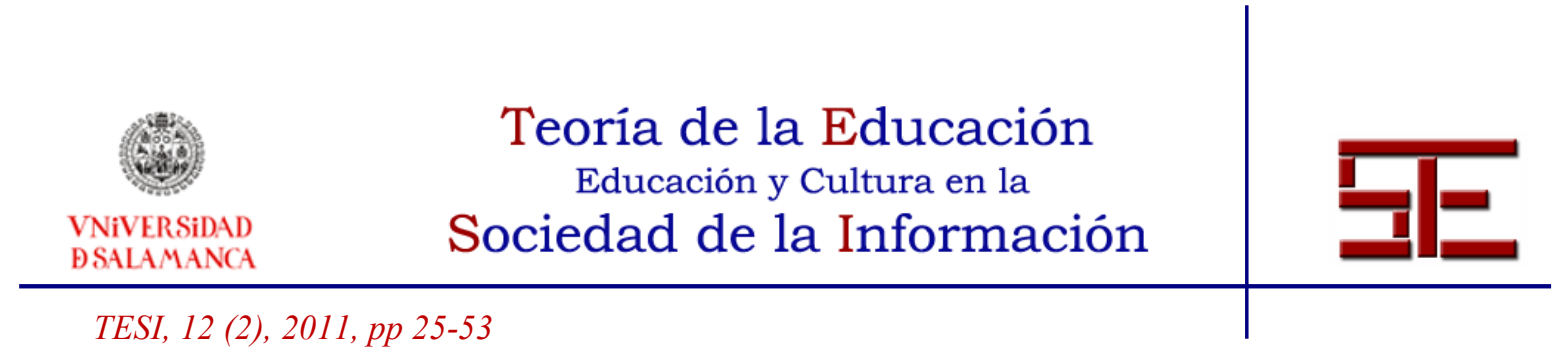

Pérez Fuentes, P. \& Andino, S. (2003). Las desigualdades de Género en el Sistema Público Universitario Vasco. Vitoria-Gasteiz: Emakunde.

Rosenbloom, J. L., Ash, R. A., Dupont, B. \& Coder, L. (2008). Why are there so few women in information technology? Assessing the role of personality in career choices. Journal of Economic Psychology, 29 (4), 543-554.

Ross, L. (2010). Computer Science: Where (and why) have all the women gone? Ponencia presentada en la Canadian Women's Studies Association Conference Saskatoon, Saskatchewan, May 27 - May 30. Extraído el 30 de marzo, 2011, de http://auspace.athabascau.ca:8080/dspace/bitstream/2149/2551/1/Lynda\%20Ros s_paper.rtf.

Royse, P., Lee, J., Undrahbuyan, B., Hopson, M. \& Consalvo, M. (2007). Women and Games: Technologies of the Gendered Self. New Media \& Society, 9 (4), 555576.

Sáinz, M., Castaño, C. \& Artal, M. (2008). Review of the concept "digital literacy" and its implications on the study of the gender digital divide. Working paper series WP08-001. Extraído el 28 de marzo, 2011, de http://www.uoc.edu/in3/dt/eng/sainz_castano_artal.pdf.

Sáinz, M., \& López-Sáez, M. (2010). Gender differences in computer attitudes and the choice of technology-related occupations in a sample of secondary students in Spain. Computers \& Education, 54, 2, 578-587.

Saloma-Akpdeonu, C. (2005). Female Spaces in the Philippines ${ }^{e e}$ ICT Industry. En C. Ng \& M. Swasti (Ed), Gender and the Digital Economy: Perspective from the Developing World (pp. 93-109). New Delhi: Sage Publications.

Sanders, J. (2006). Gender and technology: What the research tells us. En C. Skelton, B. Francis \& L. Smulyan (Eds.), Handbook of gender in education (pp. 307-322). London: Sage.

Sanz, V. (2005). Women's careers in Computer Engineering. Case study: Technical University of Madrid. Comunicación presentada en el Third European

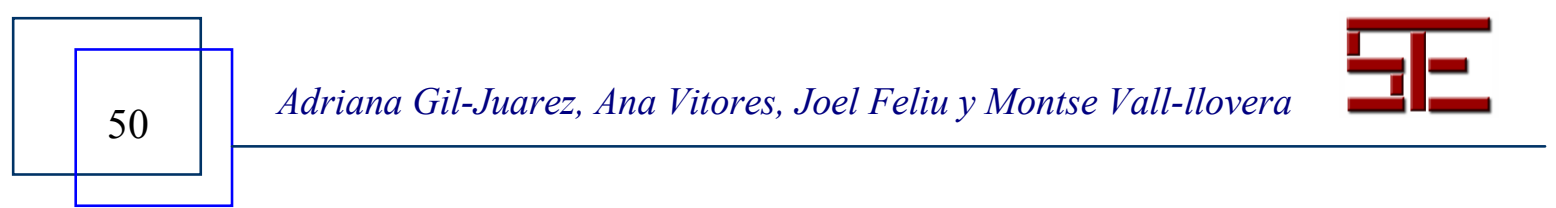




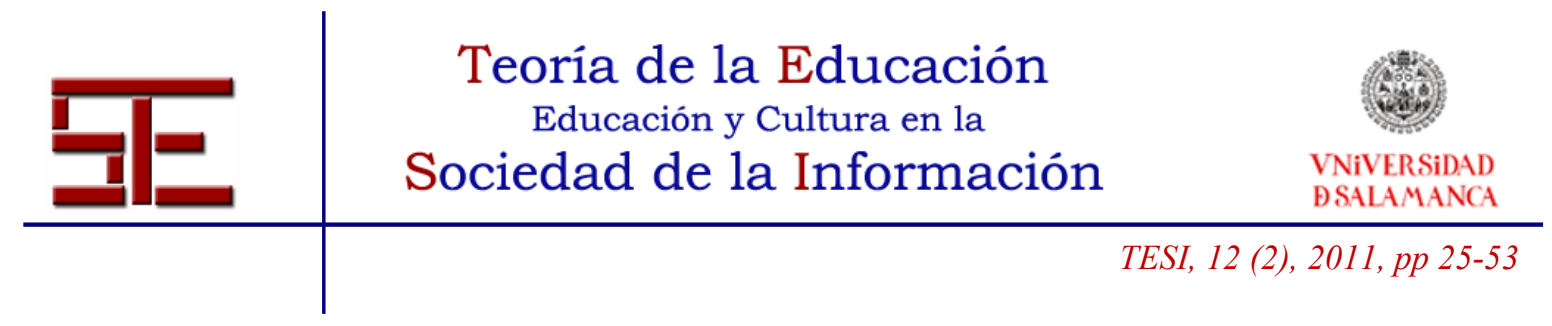

Symposium on Gender and ICT. Extraído el 15 de marzo, 2010, de http://ict.open.ac.uk/gender/2005/papers/sanz.ppt.

- (2008). Mujeres e Ingeniería Informática: el caso de la Facultad de Informática de la UPM. Arbor, 184 (733), 905-915.

Seybert, H. (2007). Gender differences in the use of computers and the Internet. Statistics in focus Luxembourg: European Communities. Extraído el 19 de marzo, 2011, de http://www.ifap.ru/library/book243.pdf.

Simard, C. \& Gilmartin, S. K. (2010). Senior Technical Women: A Profile of Success. Anita Borg Institute for Women and Technology. Extraído el 15 de marzo, 2010, de http://anitaborg.org/files/Senior-Technical-Women-A-Profile-of-Success.pdf.

Singh, K., Allen, K., Scheckler, R. \& Darlingto, L. (2007). Women in computer related majors: A critical synthesis of research and theory from 1994-2005. Review of Educational Research, 77, 500-533.

Sørensen, K. H. (2002). Love, Duty and the S-curve: An Overview of Some Current Literature on Gender and ICT ${ }^{e e}$. En K. H. Sørensen \& J. Stewart (Ed), Digital Divides and Inclusion Measures. A Review of Literature and Statistical Trends on Gender and ICT, STS Report 59 (pp. 1-36). Trondheim: NTNU, Centre for Technology and Society.

Spertus, E. (1991). Why Are There So Few Female Computer Scientists? MIT Artificial Intelligence Laboratory Technical Report number 1315. Extraído el 24 de marzo, 2011, de ftp://publications.ai.mit.edu/ai-publications/pdf/AITR-1315.pdf.

Suchman, L. (2008). Feminist STS and the Sciences of the Artificial. En E. Hackett; O. Amsterdamska; L. M \& J. Wajcman (Eds.), The Handbook of Science and Technology Studies (139-163). Cambridge, MA: MIT Press.

Todman, J. (2000). Gender differences in computer anxiety among University entrants since 1992. Computers and Education, 34, 27-35.

Turkle, S. (1988). Computational reticence: Why women fear the intimate machine. En C. Kramarae (Ed.), Technology and women's voices: Keeping in touch (pp. 4161). London: Routledge.

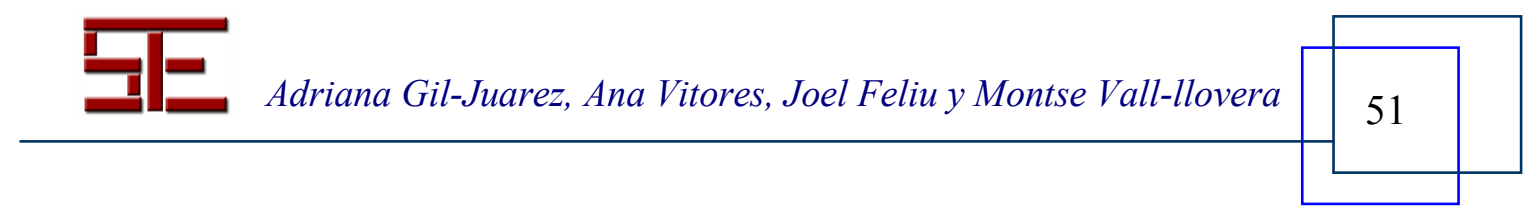




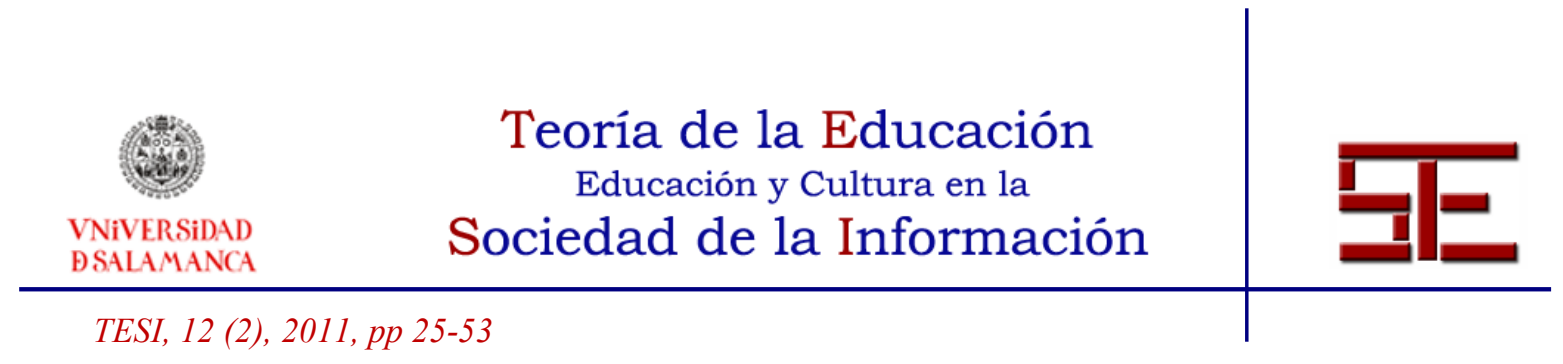

Valenduc, G., Vendramin, P., Guffens, C., Ponzellini, A. M., Lebano, A., D'Ouville, L., Collet, I., Wagner, I., Birbaumer, A., Tolar, M. \& Webster, J. (2004). Widening Women's Work in Information and Communication Technologies. European Commission (IST-2001-34520). Namur: Fondation Travail-Université.

Van Braak, J. P. (2004). Domains and determinants of university students ${ }^{\text {ee }}$ selfperceived computer competence. Computers and Education, 43 (3), 299-312.

Varma, R. \& Hahn, H. (2008). Gender and the pipeline metaphor in computing. European Journal of Engineering Education, 33 (1), 3-11.

Vekiri, I. \& Chronaki, A. (2008). Gender issues in technology use: Perceived social support, computer self-efficacy and value beliefs, and computer use beyond school. Computers \& Education, 51 (3), 1392-1404.

Vitores, A., Gil, A., Feliu, J. \& Vall-llovera, M. (2010, junio). Girls and videogames: performing femininity in a gendered site. Comunicación presentada en el BPS Social Psychology Conference: Social Psychology in Action: Theoretical Debate and Social Impact, Winchester, Reino Unido.

Volman, M., Van Eck, E., Heemskerk, I. \& Kuiper, E. (2005). New Technologies, New Differences. Gender and Ethnic Differences in Pupils' Use of ICT in Primary and Secondary Education. Computers \& Education, 45, 35-55.

Wajcman, J. (1991). Feminism Confronts Technology. Cambridge: Polity Press.

- (2006). El Tecnofeminismo. Madrid: Ediciones Cátedra.

- (2010). Feminist theories of technology. Cambridge Journal of Economics, 34 (1), 143-152.

Wajcman, J. \& Lobb, L. A. (2007). The gender relations of software work in Vietnam. Gender, Technology and Development, 11 (1), 1-26.

Walkerdine, V. (2006). Playing the game: Young girls performing femininity in video game play. Feminist Media Studies, 6 (4), 519-537.

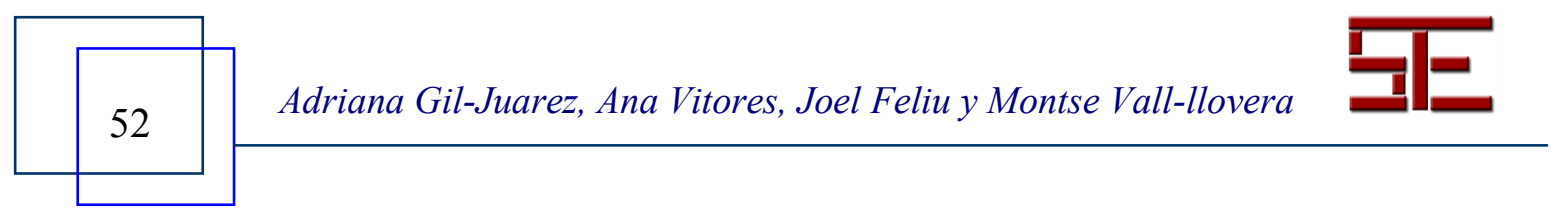




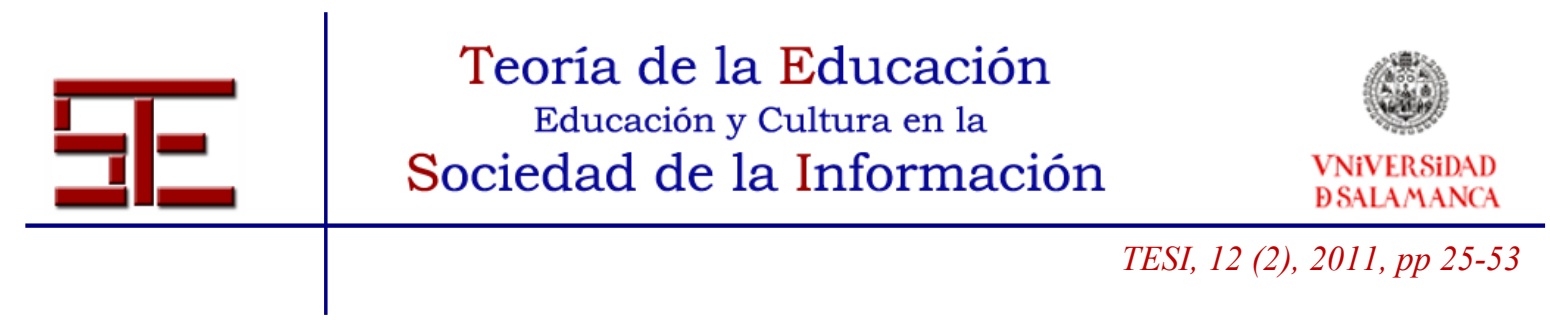

Webster, J. (2005). Women in IT Professions: Corporate Structures, Masculine Cultures. Manchester, UK: 3rd European Symposium on Gender and ICT. Working for Change.

West, C. \& Zimmerman, D. H. (1987). Doing gender. Gender \& Society, 1, 125-151.

Whitecraft, A. \& Williams, M. (2010). Why Aren't More Women in Computer Science? En Andy Oram \& Greg Wilson (Eds.), Making software (pp. 221-238). Sebastopol: O'Reilly Media, Inc.

Yelland, N., Rubin, A. \& McWilliam, E. (2002). Ghosts in the Machine: Women's Voices in Research with Technology. Peter Lang Publishing.

Para citar el presente artículo puede utilizar la siguiente referencia:

Gil-Juarez, A., Vitores, A., Feliu, J. y Vall-llovera, M. (2011). Brecha digital de género: Una revisión y una propuesta, en Barrios Vicente, I. M. (Coord.) Mujeres y la sociedad de la Información. Revista Teoría de la Educación: Educación y Cultura en la Sociedad de la Información. Vol. 12, $\mathrm{n}^{\mathrm{o}}$ 2. Universidad de Salamanca, pp. 25-53 [Fecha de consulta: $\mathrm{dd} / \mathrm{mm} /$ aaaa $]$.

http://campus.usal.es/ revistas_trabajo/index.php/revistatesi/article/view/8272/8276 